\title{
Phylogenetic relationships of cone snails endemic to Cabo Verde based on mitochondrial genomes
}

Samuel Abalde', Manuel J. Tenorio ${ }^{2}$, Carlos M. L. Afonso ${ }^{3}$, Juan E. Uribe', Ana M. Echeverry ${ }^{1}$ and Rafael Zardoya ${ }^{1 *}$ (D)

\begin{abstract}
Background: Due to their great species and ecological diversity as well as their capacity to produce hundreds of different toxins, cone snails are of interest to evolutionary biologists, pharmacologists and amateur naturalists alike. Taxonomic identification of cone snails still relies mostly on the shape, color, and banding patterns of the shell. However, these phenotypic traits are prone to homoplasy. Therefore, the consistent use of genetic data for species delimitation and phylogenetic inference in this apparently hyperdiverse group is largely wanting. Here, we reconstruct the phylogeny of the cones endemic to Cabo Verde archipelago, a well-known radiation of the group, using mitochondrial (mt) genomes.
\end{abstract}

Results: The reconstructed phylogeny grouped the analyzed species into two main clades, one including Kalloconus from West Africa sister to Trovaoconus from Cabo Verde and the other with a paraphyletic Lautoconus due to the sister group relationship of Africonus from Cabo Verde and Lautoconus ventricosus from Mediterranean Sea and neighboring Atlantic Ocean to the exclusion of Lautoconus endemic to Senegal (plus Lautoconus guanche from Mauritania, Morocco, and Canary Islands). Within Trovaoconus, up to three main lineages could be distinguished. The clade of Africonus included four main lineages (named I to IV), each further subdivided into two monophyletic groups. The reconstructed phylogeny allowed inferring the evolution of the radula in the studied lineages as well as biogeographic patterns. The number of cone species endemic to Cabo Verde was revised under the light of sequence divergence data and the inferred phylogenetic relationships.

Conclusions: The sequence divergence between continental members of the genus Kalloconus and island endemics ascribed to the genus Trovaoconus is low, prompting for synonymization of the latter. The genus Lautoconus is paraphyletic. Lautoconus ventricosus is the closest living sister group of genus Africonus. Diversification of Africonus was in allopatry due to the direct development nature of their larvae and mainly triggered by eustatic sea level changes during the Miocene-Pliocene. Our study confirms the diversity of cone endemic to Cabo Verde but significantly reduces the number of valid species. Applying a sequence divergence threshold, the number of valid species within the sampled Africonus is reduced to half.

Keywords: Mitochondrial genomes, Africonus, Trovaoconus, Kalloconus

\footnotetext{
* Correspondence: rafaz@mncn.csic.es

${ }^{1}$ Museo Nacional de Ciencias Naturales (MNCN-CSIC), José Gutiérrez Abascal 2, 28006 Madrid, Spain

Full list of author information is available at the end of the article
} 


\section{Background}

The cone snails (Conidae, Gastropoda) endemic to the archipelago of Cabo Verde in West Africa represent one of the few textbook examples of a well-documented insular species radiation involving marine organisms [1-3]. Cone snails, which are found in tropical and subtropical marine waters throughout the world, show a hotspot of species diversity in the Cabo Verde archipelago with up to 95 endemic species (roughly 10\% of cone species diversity worldwide) narrowly confined to about $4000 \mathrm{~km}^{2}$ [4]. As in other parts of the world, cone snails endemic to Cabo Verde constitute a key component of the intertidal and subtidal ecosystems associated to rocky shores, coral reefs, and sandy bottoms. All cones endemic to Cabo Verde feed on marine annelid worms [1] and use a sophisticated venom apparatus (including a venom gland that produces conotoxins and a specialized harpoon-like radular tooth) to capture their preys [5]. Another interesting biological feature common to all these endemic species is that they have direct development. Their larvae lack a pelagic stage, and thus show a considerably reduced dispersal capacity [1]. Survival rate is higher for this type of larvae since they are less likely to be eaten by predators and are not dependent on plankton for feeding (i.e, non-planktotrophic).

The origin and evolutionary history of cones endemic to Cabo Verde has been the subject of several recent phylogenetic studies [1, 2, 6, 7]. Molecular phylogenies demonstrated that two different ancestors reached the archipelago independently and subsequently diversified following recurrent biogeographic patterns [1, 2, 7]. The existence of two clades led to the classification of cone species endemic to Cabo Verde into two genera, Africonus and Trovaoconus [8]. The question of which species are the closest living sister groups to Africonus and Trovaoconus remains open $[1,2]$. According to a previous study, the ancestor of Africonus colonized the archipelago in the Miocene, about 16.5 million years ago (mya; [1]), and spread to all islands (except Fogo, the youngest, with steep slopes in the coast and ongoing volcanic activity). Most (95\%) of the currently described species endemic to Cabo Verde belong to Africonus, and are normally referred to as restricted to a single island and in some cases even to single bays within an island [3]. The ancestor of Trovaoconus arrived at Cabo Verde archipelago in the Pliocene, about 4.6 mya, and diversified only in four islands (Sal, Boa Vista, Maio, and possibly Santiago), which are the closest to the continent [1]. These cones are significantly larger in size than those belonging to Africonus and show wider distributions extending in some cases to more than one island. It has been hypothesized that diversification within each genus was in allopatry and followed recurrent eustatic sea level changes during the Neogene that intermittently connected and disconnected the islands [1, 7]. However, sea level fluctuations alone do not fully explain the extraordinary diversity of cones in Cape
Verde since nearby archipelagos in the Macaronesia biogeographic region such as the Canary Islands subjected to similar trends since the Miocene do not have endemic cone species [6]. A larger distance to the mainland, which enhances isolation and restricts gene flow combined with a higher mean sea surface temperature and the presence of more suitable habitats may have promoted a significant increase in diversification rates in the Cabo Verde archipelago [6].

The rate of description of new cone species endemic to Cabo Verde has accelerated more than expected during the last years (Fig. 1). After the early descriptions in the eighteenth and nineteenth centuries based on samples brought to Europe by naturalists [9], the main contribution to the cataloguing of cone species endemic to Cabo Verde was due to the work of Emilio Rolán [10], who drew attention to this singular radiation. Hence, around year 2000, there were about 50 species recognized [11] and remarkably this number has almost doubled in the last 2-3 years [12-19]. However, it is important to note that many of the recent species diagnoses in cones are mainly based on the shape, color, and banding patterns of the shell. These phenotypic characters are highly variable at the population level and prone to local adaptation and convergence, making species assignment problematic and sometimes, misleading [7]. In many cases, distinguishing whether different shell morphotypes of cone snails represent valid species or ecotypes of the same species is challenging [20]. Therefore, determination of genetic variation and inference of phylogenetic relationships based on DNA sequence data are timely as part of a multidisciplinary approach [21] to identify and delimit species and to understand evolutionary processes underlying diversification within cones, in general, and within those endemic to Cabo Verde, in particular.

Here, we used nearly complete mitochondrial (mt) genomes, which have proven to successfully reconstruct robust phylogenies of Conidae [22] and of particular groups such as the cones endemic to Senegal [23]. In this study, we sequenced the nearly complete mt genomes of 88 individuals representing different populations and species of Africonus and Trovaoconus endemic to Cabo Verde. We aimed to: (1) reconstruct a highly resolved phylogeny of cones endemic to Cabo Verde; (2) determine the closest living sister groups of Africonus and Trovaoconus; (3) date major cladogenetic events and analyze biogeographical patterns; (4) study radular tooth evolution within the two genera; and (5) provide a first genetic hypothesis of species delimitation in the radiation of Cabo Verde endemic cones.

\section{Results}

Sequencing, assembly, and genome organization

The nucleotide sequences of the near-complete $\mathrm{mt}$ genomes of 75 specimens of Africonus, 13 specimens of 


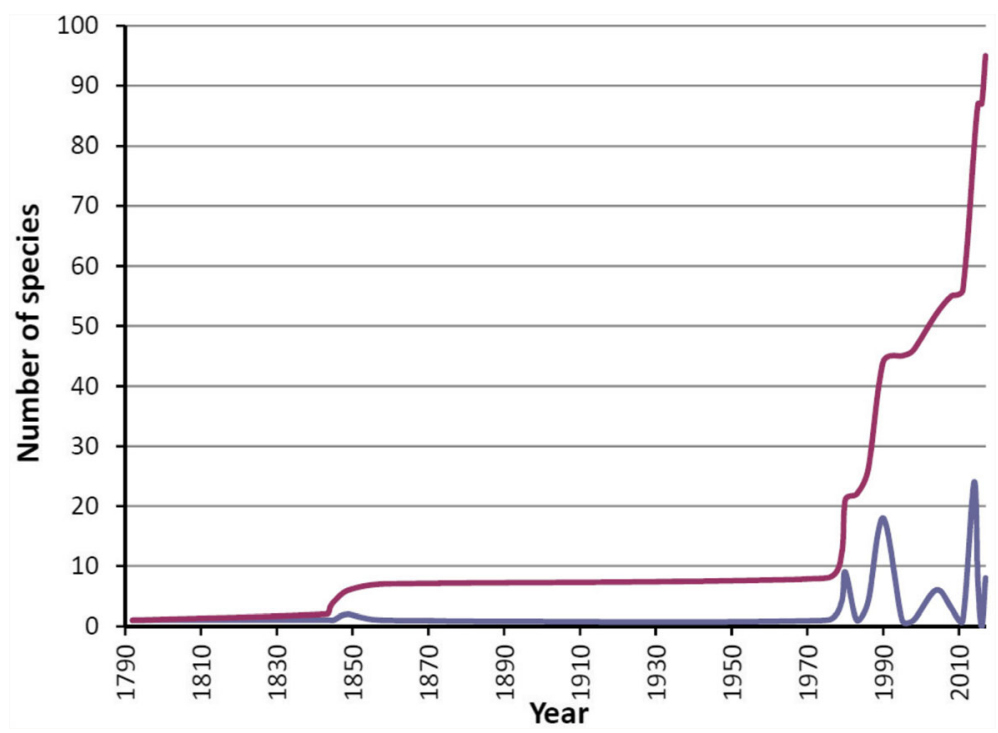

Fig. 1 Number (blue) and accumulated number (red) of cone species described for the Cabo Verde archipelago per year

Trovaoconus, and one specimen of Lautoconus ventricosus were determined (Table 1). These mt genomes lacked the $\operatorname{trn} F$ gene, the control region, and the start of the cox3 gene because the corresponding fragment was not PCR amplified. The number of reads, mean coverage, and length of each $\mathrm{mt}$ genome are provided in Table 1. The mt genomes of Africonus boavistensis and Africonus denizi received the minimum $(42,021)$ and maximum $(906,765)$ number of reads, respectively. The same samples received the minimum $(412 \times)$ and maximum $(8,885 \times)$ mean coverage, respectively (Table 1$)$. All sequenced $\mathrm{mt}$ genomes encode for 13 proteincoding, 2 rRNA and 21 tRNA genes (but note that the trnF gene could not be determined; see above). They all share the same genome organization: the major strand encodes all genes, except those forming the cluster MYCWQGE (trnM, trn $Y, \operatorname{trn} C, \operatorname{trn} W, \operatorname{trn} Q, \operatorname{trn} G, \operatorname{trn} E)$ and the trnT gene.

\section{Phylogenetic relationships and sequence divergences between clades}

Phylogenetic relationships of cones endemic to Cabo Verde were reconstructed based on the nucleotide sequences of the concatenated $13 \mathrm{mt}$ protein-coding and two rRNA genes using probabilistic methods and Chelyconus ermineus as outgroup. The final matrix was 13,572 positions in length. According to the AIC, the best partition scheme for the protein-coding genes was the one combining all these genes but analyzing each codon position separately. The best substitution model for each of the three codon positions was GTR $+\mathrm{I}+\mathrm{G}$. For the rRNA genes, the best scheme had both genes combined under the GTR $+\mathrm{I}+\mathrm{G}$ model. Both, ML $(-\ln L=$
$75,600.18)$ and BI $(-\ln L=76,002.71$ for run $1 ;-\ln L=$ $76,288.44$ for run 2) arrived at almost identical topology (Figs. 2 and 3). Most nodes received high statistical support and differences in topology were restricted exclusively to three relatively shallow nodes that had low support in ML and were unresolved in BI. Two of these nodes involved almost identical sequences and corresponded to Africonus bernardinoil Africonus pseudocuneolus and Africonus teodorael Africonus fiadeiroi, respectively. The third unresolved node corresponded to a trichotomy involving Africonus felitae, Africonus regonae and Africonus longilineus/ Africonus cagarralensis/Africonus melissae.

The reconstructed phylogeny (Fig. 2) grouped the analyzed species into two main clades, one including Kalloconus from mainland West Africa sister to Trovaoconus from Cabo Verde and the other having paraphyletic Lautoconus due to the sister group relationship of Africonus from Cabo Verde and Lautoconus ventricosus from Mediterranean Sea and neighboring Atlantic Ocean to the exclusion of Lautoconus endemic to Senegal (plus Lautoconus guanche from Mauritania, Morocco, and Canary Islands). Within Trovaoconus, up to three main lineages could be distinguished (Fig. 2). The first one included two specimens from Sal initially identified as Trovaoconus ateralbus, which were sister to a clade including one lineage with specimens from Maio and Boa Vista identified as Trovaoconus venulatus and another lineage having mostly specimens of Trovaoconus pseudonivifer from Maio and Boa Vista but also one specimen of Trovaoconus trochulus from Boa Vista and one of Trovaoconus atlanticoselvagem from Baixo João Valente (Fig. 2).

The clade of Africonus from Cabo Verde included four main lineages (named I to IV), each further subdivided 
Table 1 Mitochondrial (mt) genomes analyzed in this study

\begin{tabular}{|c|c|c|c|c|c|c|c|c|c|c|}
\hline \multicolumn{11}{|c|}{ New mt genomes } \\
\hline \multirow[t]{2}{*}{$\overline{\mathrm{IDCV}}$} & \multirow{2}{*}{$\begin{array}{l}\text { Initial species } \\
\text { identification }\end{array}$} & \multirow[t]{2}{*}{ Location } & \multirow[t]{2}{*}{ Coordinates } & \multicolumn{2}{|c|}{ Coverage } & \multirow{2}{*}{$\begin{array}{l}\text { Length } \\
\text {-(bp) }\end{array}$} & \multirow{2}{*}{$\begin{array}{l}\text { GenBank } \\
\text { Acc. No }\end{array}$} & \multirow{2}{*}{$\begin{array}{l}\text { Voucher DNA } \\
\text { (MNCN/ADN) }\end{array}$} & \multirow{2}{*}{$\begin{array}{l}\text { Voucher shell } \\
\text { (MNCN 15.05/) }\end{array}$} & \multirow{2}{*}{$\begin{array}{l}\text { New species } \\
\text { proposed }^{\mathrm{a}}\end{array}$} \\
\hline & & & & $\begin{array}{l}\mathrm{n}^{\circ} \\
\text { reads }\end{array}$ & $\begin{array}{l}\text { mean } \\
\text { depth }\end{array}$ & & & & & \\
\hline 1020 & $\begin{array}{l}\text { Africonus } \\
\text { antoniaensis }\end{array}$ & $\begin{array}{l}\text { Água Doce, Boa Vista, } \\
\text { Cabo Verde }\end{array}$ & $\begin{array}{l}16^{\circ} 12^{\prime} 29^{\prime \prime} \mathrm{N} \\
22^{\circ} 44^{\prime} 7^{\prime \prime} \mathrm{W}\end{array}$ & 151104 & 1476.8 & 15332 & MF491587 & 95072 & 79889 & - \\
\hline 0885 & $\begin{array}{l}\text { Africonus } \\
\text { antoniomonteiroi }\end{array}$ & $\begin{array}{l}\text { Pedra Lume, Sal, Cabo } \\
\text { Verde }\end{array}$ & $\begin{array}{l}16^{\circ} 45^{\prime} 44^{\prime \prime} \mathrm{N} \\
22^{\circ} 53^{\prime} 2^{\prime \prime} \mathrm{W}\end{array}$ & 232069 & 2273.4 & 15328 & MF491578 & 95063 & 79794 & - \\
\hline 0927 & $\begin{array}{l}\text { Africonus } \\
\text { bernardinoi }\end{array}$ & $\begin{array}{l}\text { Pedra Lume, Sal, Cabo } \\
\text { Verde }\end{array}$ & $\begin{array}{l}16^{\circ} 45^{\prime} 44^{\prime \prime} \mathrm{N} \\
22^{\circ} 53^{\prime} 2^{\prime \prime} \mathrm{W}\end{array}$ & 59799 & 583.3 & 15328 & MF491582 & 95067 & 79835 & $\begin{array}{l}\text { Africonus } \\
\text { cuneolus }\end{array}$ \\
\hline 0520 & $\begin{array}{l}\text { Africonus } \\
\text { boavistensis }\end{array}$ & $\begin{array}{l}\text { Baía do Ervatão (North), } \\
\text { Boa Vista, Cabo Verde }\end{array}$ & $\begin{array}{l}16^{\circ} 12^{\prime} 3^{\prime \prime} \mathrm{N} \\
22^{\circ} 54^{\prime} 43^{\prime \prime} \mathrm{W}\end{array}$ & 42021 & 412.8 & 15217 & MF491563 & 95045 & 80413 & - \\
\hline 1135 & Africonus cabraloi & $\begin{array}{l}\text { Estancinha, Boa Vista, } \\
\text { Cabo Verde }\end{array}$ & $\begin{array}{l}16^{\circ} 13^{\prime} 12^{\prime \prime} \mathrm{N} \\
22^{\circ} 55^{\prime} 9^{\prime \prime} \mathrm{W}\end{array}$ & 74446 & 730.4 & 15329 & MF491598 & 95083 & 80004 & $\begin{array}{l}\text { Africonus } \\
\text { crotchii }\end{array}$ \\
\hline 0895 & $\begin{array}{l}\text { Africonus } \\
\text { cagarralensis }\end{array}$ & $\begin{array}{l}\text { Pedra Lume, Sal, Cabo } \\
\text { Verde }\end{array}$ & $\begin{array}{l}16^{\circ} 45^{\prime} 44 " \mathrm{~N}, \\
22^{\circ} 53^{\prime} 2 \mathrm{~W}\end{array}$ & 161290 & 1367.2 & 15320 & MF491579 & 95064 & 79804 & $\begin{array}{l}\text { Africonus } \\
\text { longilineus }\end{array}$ \\
\hline 0173 & $\begin{array}{l}\text { Africonus } \\
\text { calhetae }\end{array}$ & $\begin{array}{l}\text { Praia da Soca, Maio, } \\
\text { Cabo Verde }\end{array}$ & $\begin{array}{l}15^{\circ} 15^{\prime} 8^{\prime \prime} \mathrm{N} \\
23^{\circ} 13^{\prime} 4^{\prime \prime} \mathrm{W}\end{array}$ & 55433 & 544.7 & 15242 & MF491534 & 95016 & 78798 & - \\
\hline 0920 & $\begin{array}{l}\text { Africonus cf. } \\
\text { anthonyi }\end{array}$ & $\begin{array}{l}\text { Ilhéus do Chano, Sal, } \\
\text { Cabo Verde }\end{array}$ & $\begin{array}{l}16^{\circ} 41^{\prime} 37^{\prime \prime} \mathrm{N} \\
22^{\circ} 52^{\prime} 47^{\prime \prime} \mathrm{W}\end{array}$ & 172336 & 1678.6 & 15315 & MF491581 & 95066 & 79828 & $\begin{array}{l}\text { Africonus } \\
\text { cuneolus }\end{array}$ \\
\hline 0162 & $\begin{array}{l}\text { Africonus cf. } \\
\text { claudiae }\end{array}$ & $\begin{array}{l}\text { Praia da Soca, Maio, } \\
\text { Cabo Verde }\end{array}$ & $\begin{array}{l}15^{\circ} 15^{\prime} 8^{\prime \prime} \mathrm{N} \\
23^{\circ} 13^{\prime} 4^{\prime \prime} \mathrm{W}\end{array}$ & 87407 & 858.6 & 15326 & MF491533 & 95015 & 78787 & $\begin{array}{l}\text { Africonus } \\
\text { calhetae }\end{array}$ \\
\hline 0465 & $\begin{array}{l}\text { Africonus cf. } \\
\text { delanoyae }\end{array}$ & $\begin{array}{l}\text { Ponta Antónia, Boa Vista, } \\
\text { Cabo Verde }\end{array}$ & $\begin{array}{l}16^{\circ} 13^{\prime} 24^{\prime \prime} \mathrm{N} \\
22^{\circ} 46^{\prime} 59^{\prime \prime} \mathrm{W}\end{array}$ & 382817 & 3736.1 & 15335 & MF491559 & 95041 & 80409 & $\begin{array}{l}\text { Africonus } \\
\text { fuscoflavus }\end{array}$ \\
\hline 0207 & $\begin{array}{l}\text { Africonus cf. } \\
\text { galeao }\end{array}$ & $\begin{array}{l}\text { Ponta Pipa, Maio, Cabo } \\
\text { Verde }\end{array}$ & $\begin{array}{l}15^{\circ} 19^{\prime} 30^{\prime \prime} \mathrm{N} \\
23^{\circ} 9^{\prime} 48^{\prime \prime} \mathrm{W}\end{array}$ & 81447 & 797.3 & 15325 & MF491536 & 95018 & 78832 & $\begin{array}{l}\text { Africonus } \\
\text { galeao }\end{array}$ \\
\hline 0135 & $\begin{array}{l}\text { Africonus cf. } \\
\text { gonsaloi }\end{array}$ & $\begin{array}{l}\text { Praia Gonçalo, Maio, } \\
\text { Cabo Verde }\end{array}$ & $\begin{array}{l}15^{\circ} 16^{\prime} 13^{\prime \prime} \mathrm{N} \\
23^{\circ} 6^{\prime} 15^{\prime \prime} \mathrm{W}\end{array}$ & 148032 & 1455.5 & 15250 & MF491529 & 95011 & 78760 & $\begin{array}{l}\text { Africonus } \\
\text { gonsaloi }\end{array}$ \\
\hline 0380 & $\begin{array}{l}\text { Africonus cf. } \\
\text { miguelfiaderoi }\end{array}$ & $\begin{array}{l}\text { Jorrita, Baía da Gata, Boa } \\
\text { Vista, Cabo Verde }\end{array}$ & $\begin{array}{l}16^{\circ} 12^{\prime} 9^{\prime \prime} \mathrm{N} \\
22^{\circ} 42^{\prime} 22^{\prime \prime} \mathrm{W}\end{array}$ & 358342 & 3507.9 & 15328 & MF491548 & 95030 & 80398 & $\begin{array}{l}\text { Africonus } \\
\text { vulcanus }\end{array}$ \\
\hline 1400 & $\begin{array}{l}\text { Africonus cf. } \\
\text { miruchae }\end{array}$ & $\begin{array}{l}\text { Calhau, São Vicente, } \\
\text { Cabo Verde }\end{array}$ & $\begin{array}{l}16^{\circ} 51^{\prime} 7 " \mathrm{~N}, \\
24^{\circ} 51^{\prime} 59^{\prime \prime} \mathrm{W}\end{array}$ & 523002 & 5104.9 & 15321 & MF491601 & 95088 & 78562 & $\begin{array}{l}\text { Africonus sp. } \\
\text { nov. } 1\end{array}$ \\
\hline 0223 & $\begin{array}{l}\text { Africonus } \\
\text { claudiae }\end{array}$ & $\begin{array}{l}\text { Ponta Pipa, Maio, Cabo } \\
\text { Verde }\end{array}$ & $\begin{array}{l}15^{\circ} 19^{\prime} 30^{\prime \prime} \mathrm{N} \\
23^{\circ} 9^{\prime} 48^{\prime \prime} \mathrm{W}\end{array}$ & 148508 & 1434.5 & 15337 & MF491537 & 95019 & 78848 & $\begin{array}{l}\text { Africonus } \\
\text { galeao }\end{array}$ \\
\hline 0303 & Africonus condei & $\begin{array}{l}\text { Baía Grande, Derrubado, } \\
\text { Boa Vista, Cabo Verde }\end{array}$ & $\begin{array}{l}16^{\circ} 13^{\prime} 31^{\prime \prime N}, \\
22^{\circ} 47^{\prime} 17^{\prime \prime} \mathrm{W}\end{array}$ & 253863 & 2472.2 & 15248 & MF491542 & 95024 & 80392 & $\begin{array}{l}\text { Africonus } \\
\text { crotchii }\end{array}$ \\
\hline 0045 & Africonus crioulus & $\begin{array}{l}\text { Praia Santana, Maio, } \\
\text { Cabo Verde }\end{array}$ & $\begin{array}{l}15^{\circ} 18^{\prime} 13^{\prime \prime} \mathrm{N} \\
23^{\circ} 11^{\prime} 49^{\prime \prime} \mathrm{W}\end{array}$ & 255019 & 2502 & 15247 & MF491521 & 95003 & 78670 & $\begin{array}{l}\text { Africonus } \\
\text { maioensis }\end{array}$ \\
\hline 1075 & Africonus crotchii & $\begin{array}{l}\text { Morro de Areia, Boa } \\
\text { Vista, Cabo Verde }\end{array}$ & $\begin{array}{l}16^{\circ} 5^{\prime} 24^{\prime \prime} \mathrm{N} \\
22^{\circ} 57^{\prime} 7 " \mathrm{~W}\end{array}$ & 332385 & 3237.6 & 15329 & MF491591 & 95076 & 79944 & - \\
\hline 0803 & $\begin{array}{l}\text { Africonus } \\
\text { cuneolus }\end{array}$ & $\begin{array}{l}\text { Calheta Funda, Sal, Cabo } \\
\text { Verde }\end{array}$ & $\begin{array}{l}16^{\circ} 39^{\prime} 6^{\prime \prime} \mathrm{N} \\
22^{\circ} 56^{\prime} 53^{\prime \prime} \mathrm{W}\end{array}$ & 184181 & 1791.6 & 15329 & MF491569 & 95053 & 79712 & - \\
\hline 0936 & $\begin{array}{l}\text { Africonus } \\
\text { cuneolus }\end{array}$ & $\begin{array}{l}\text { Santa Maria, Sal, Cabo } \\
\text { Verde }\end{array}$ & $\begin{array}{l}16^{\circ} 35^{\prime} 38^{\prime \prime} \mathrm{N} \\
22^{\circ} 53^{\prime} 36^{\prime \prime} \mathrm{W}\end{array}$ & 80472 & 787.4 & 15328 & MF491583 & 95068 & 79844 & - \\
\hline 1420 & $\begin{array}{l}\text { Africonus } \\
\text { curralensis }\end{array}$ & $\begin{array}{l}\text { Praia de Palmo Tostão, } \\
\text { Santa Luzia, Cabo Verde }\end{array}$ & $\begin{array}{l}16^{\circ} 45^{\prime} 19^{\prime \prime} \mathrm{N} \\
24^{\circ} 45^{\prime} 24^{\prime \prime} \mathrm{W}\end{array}$ & 857123 & 8358.6 & 15329 & MF491602 & 95089 & 78581 & - \\
\hline 1017 & Africonus damioi & $\begin{array}{l}\text { Água Doce, Boa Vista, } \\
\text { Cabo Verde }\end{array}$ & $\begin{array}{l}16^{\circ} 12^{\prime} 29^{\prime \prime N}, \\
22^{\circ} 44^{\prime} 7 " \mathrm{~W}\end{array}$ & 76477 & 745.5 & 15326 & MF491586 & 95071 & 79886 & $\begin{array}{l}\text { Africonus } \\
\text { roeckeli }\end{array}$ \\
\hline 0405 & $\begin{array}{l}\text { Africonus } \\
\text { damottai }\end{array}$ & $\begin{array}{l}\text { Baía da Gata (center), } \\
\text { Boa Vista, Cabo Verde }\end{array}$ & $\begin{array}{l}16^{\circ} 11^{\prime} 50^{\prime \prime} \mathrm{N} \\
22^{\circ} 42^{\prime} 32^{\prime \prime} \mathrm{W}\end{array}$ & 315488 & 2914.3 & 15358 & MF491551 & 95033 & 80401 & - \\
\hline 1428 & $\begin{array}{l}\text { Africonus } \\
\text { decoratus }\end{array}$ & $\begin{array}{l}\text { Curral, Santa Luzia, Cabo } \\
\text { Verde }\end{array}$ & $\begin{array}{l}16^{\circ} 46^{\prime} 23^{\prime \prime} \mathrm{N} \\
24^{\circ} 47^{\prime} 13^{\prime \prime} \mathrm{W}\end{array}$ & 566822 & 5540.2 & 15326 & MF491603 & 95090 & 78589 & - \\
\hline 0370 & $\begin{array}{l}\text { Africonus } \\
\text { delanoyae }\end{array}$ & $\begin{array}{l}\text { Jorrita, Baía da Gata, Boa } \\
\text { Vista, Cabo Verde }\end{array}$ & $\begin{array}{l}16^{\circ} 12^{\prime} 9^{\prime \prime} \mathrm{N} \\
22^{\circ} 42^{\prime} 22^{\prime \prime} \mathrm{W}\end{array}$ & 158489 & 1543.7 & 15323 & MF491547 & 95029 & 80397 & - \\
\hline 1471 & Africonus denizi & $\begin{array}{l}\text { Praia Grande, São } \\
\text { Vicente, Cabo Verde }\end{array}$ & $\begin{array}{l}16^{\circ} 51^{\prime} 40^{\prime \prime} \mathrm{N} \\
24^{\circ} 52^{\prime} 30^{\prime \prime} \mathrm{W}\end{array}$ & 906765 & 8885.2 & 15326 & MF491605 & 95092 & 78621 & - \\
\hline 0315 & & & & 214173 & 2089.2 & 15243 & MF491543 & 95025 & 80393 & \\
\hline
\end{tabular}


Table 1 Mitochondrial (mt) genomes analyzed in this study (Continued)

\begin{tabular}{|c|c|c|c|c|c|c|c|c|c|c|}
\hline & $\begin{array}{l}\text { Africonus } \\
\text { derrubado }\end{array}$ & $\begin{array}{l}\text { Baía Grande, Derrubado, } \\
\text { Boa Vista, Cabo Verde }\end{array}$ & $\begin{array}{l}16^{\circ} 13^{\prime} 31 " \mathrm{~N}, \\
22^{\circ} 47^{\prime} 17^{\prime \prime W}\end{array}$ & & & & & & & $\begin{array}{l}\text { Africonus } \\
\text { damottai }\end{array}$ \\
\hline 0565 & $\begin{array}{l}\text { Africonus } \\
\text { diminutus }\end{array}$ & $\begin{array}{l}\text { Ilhéu de Sal Rei, Boa } \\
\text { Vista, Cabo Verde }\end{array}$ & $\begin{array}{l}16^{\circ} 9^{\prime} 50^{\prime \prime} \mathrm{N} \\
22^{\circ} 55^{\prime} 31^{\prime \prime} \mathrm{W}\end{array}$ & 840424 & 8204.1 & 15330 & MF491566 & 95049 & 80416 & - \\
\hline 1025 & $\begin{array}{l}\text { Africonus } \\
\text { docensis }\end{array}$ & $\begin{array}{l}\text { Água Doce, Boa Vista, } \\
\text { Cabo Verde }\end{array}$ & $\begin{array}{l}16^{\circ} 12^{\prime} 29^{\prime \prime} \mathrm{N} \\
22^{\circ} 44^{\prime} 7 \mathrm{~W}\end{array}$ & 47313 & 464.8 & 15329 & MF491588 & 95073 & 79894 & $\begin{array}{l}\text { Africonus } \\
\text { crotchii }\end{array}$ \\
\hline 0385 & Africonus evorai & $\begin{array}{l}\text { Zebraca (near Ilhéu do } \\
\text { Galeão), Boa Vista, Cabo } \\
\text { Verde }\end{array}$ & $\begin{array}{l}16^{\circ} 12^{\prime} 6^{\prime \prime} \mathrm{N} \\
22^{\circ} 42^{\prime} 40^{\prime \prime} \mathrm{W}\end{array}$ & 226416 & 2218 & 15243 & MF491549 & 95031 & 80399 & $\begin{array}{l}\text { Africonus } \\
\text { crotchii }\end{array}$ \\
\hline 0070 & $\begin{array}{l}\text { Africonus } \\
\text { fantasmalis }\end{array}$ & $\begin{array}{l}\text { Porto Cais, Maio, Cabo } \\
\text { Verde }\end{array}$ & $\begin{array}{l}15^{\circ} 19^{\prime} 15^{\prime \prime} \mathrm{N} \\
23^{\circ} 11^{\prime} 10^{\prime \prime} \mathrm{W}\end{array}$ & 97527 & 954.7 & 15330 & MF491524 & 95006 & 78695 & $\begin{array}{l}\text { Africonus } \\
\text { fuscoflavus }\end{array}$ \\
\hline 0835 & Africonus felitae & $\begin{array}{l}\text { Rabo de Junco, Sal, Cabo } \\
\text { Verde }\end{array}$ & $\begin{array}{l}16^{\circ} 41^{\prime} 44^{\prime \prime} \mathrm{N} \\
22^{\circ} 58^{\prime} 35^{\prime \prime} \mathrm{W}\end{array}$ & 344190 & 3343.9 & 15404 & MF491573 & 95057 & 79744 & - \\
\hline 1437 & $\begin{array}{l}\text { Africonus } \\
\text { fernandesi }\end{array}$ & $\begin{array}{l}\text { Porto Novo, Santo } \\
\text { Antão, Cabo Verde }\end{array}$ & $\begin{array}{l}17^{\circ} 1^{\prime} 4^{\prime \prime} \mathrm{N} \\
25^{\circ} 3^{\prime} 22^{\prime \prime} \mathrm{W}\end{array}$ & 742414 & 7244.2 & 15324 & MF491604 & 95091 & 78598 & - \\
\hline 0332 & Africonus fiadeiroi & $\begin{array}{l}\text { Derrubado (bay West), } \\
\text { Boa Vista, Cabo Verde }\end{array}$ & $\begin{array}{l}16^{\circ} 13^{\prime} 22^{\prime \prime} \mathrm{N} \\
22^{\circ} 47^{\prime} 41^{\prime \prime} \mathrm{W}\end{array}$ & 205910 & 2016.5 & 15243 & MF491545 & 95027 & 80395 & $\begin{array}{l}\text { Africonus } \\
\text { crotchii }\end{array}$ \\
\hline 0855 & $\begin{array}{l}\text { Africonus } \\
\text { fontonae }\end{array}$ & $\begin{array}{l}\text { Baía da Fontona, Sal, } \\
\text { Cabo Verde }\end{array}$ & $\begin{array}{l}16^{\circ} 44^{\prime} 22^{\prime \prime} \mathrm{N} \\
22^{\circ} 58^{\prime} 46^{\prime \prime} \mathrm{W}\end{array}$ & 156259 & 1523.9 & 15328 & MF491575 & 95059 & 79764 & $\begin{array}{l}\text { Africonus } \\
\text { cuneolus }\end{array}$ \\
\hline 0945 & $\begin{array}{l}\text { Africonus } \\
\text { fontonae }\end{array}$ & Regona, Sal, Cabo Verde & $\begin{array}{l}16^{\circ} 48^{\prime} 5^{\prime \prime} \mathrm{N} \\
22^{\circ} 59^{\prime} 33^{\prime \prime} \mathrm{W}\end{array}$ & 56310 & 549.8 & 15327 & MF491584 & 95069 & 79853 & $\begin{array}{l}\text { Africonus } \\
\text { regonae }\end{array}$ \\
\hline 0450 & $\begin{array}{l}\text { Africonus } \\
\text { fuscoflavus }\end{array}$ & $\begin{array}{l}\text { Derrubado (bay East), } \\
\text { Boa Vista, Cabo Verde }\end{array}$ & $\begin{array}{l}16^{\circ} \\
13^{\prime} 331^{\prime \prime} \mathrm{N} \\
22^{\circ} 47^{\prime} 3^{\prime \prime} \mathrm{W}\end{array}$ & 151904 & 1478.6 & 15331 & MF491557 & 95039 & 80407 & - \\
\hline 0052 & Africonus galeao & $\begin{array}{l}\text { Navio Quebrado, Terras } \\
\text { Salgadas, Maio, Cabo } \\
\text { Verde }\end{array}$ & $\begin{array}{l}15^{\circ} 18^{\prime} 54^{\prime \prime} \mathrm{N} \\
23^{\circ} 11^{\prime 2} 2^{\mathrm{W}}\end{array}$ & 117940 & 1139.2 & 15326 & MF491522 & 95004 & 78677 & - \\
\hline 0134 & $\begin{array}{l}\text { Africonus } \\
\text { gonsaloi }\end{array}$ & $\begin{array}{l}\text { Praia Gonçalo, Maio, } \\
\text { Cabo Verde }\end{array}$ & $\begin{array}{l}15^{\circ} 16^{\prime} 13^{\prime \prime N}, \\
23^{\circ} 6^{\prime} 15^{\prime \prime} \mathrm{W}\end{array}$ & 188174 & 1835.9 & 15339 & MF491528 & 95010 & 78759 & - \\
\hline 1390 & $\begin{array}{l}\text { Africonus } \\
\text { grahami }\end{array}$ & $\begin{array}{l}\text { Calhau, São Vicente, } \\
\text { Cabo Verde }\end{array}$ & $\begin{array}{l}16^{\circ} 51^{\prime} 7 " \mathrm{~N} \\
24^{\circ} 51^{\prime} 59^{\prime \prime} \mathrm{W}\end{array}$ & 464704 & 4536.1 & 15325 & MF491599 & 95086 & 78552 & - \\
\hline 0140 & $\begin{array}{l}\text { Africonus } \\
\text { irregularis }\end{array}$ & $\begin{array}{l}\text { Porto Cais (North), Maio, } \\
\text { Cabo Verde }\end{array}$ & $\begin{array}{l}15^{\circ} 19^{\prime} 45^{\prime \prime N} \\
23^{\circ} 10^{\prime} 57^{\prime \prime} \mathrm{W}\end{array}$ & 202254 & 1937.9 & 15321 & MF491530 & 95012 & 78765 & $\begin{array}{l}\text { Africonus } \\
\text { maioensis }\end{array}$ \\
\hline 0317 & $\begin{array}{l}\text { Africonus } \\
\text { irregularis }\end{array}$ & $\begin{array}{l}\text { Baía Grande, Derrubado, } \\
\text { Boa Vista, Cabo Verde }\end{array}$ & $\begin{array}{l}16^{\circ} 13^{\prime} 31^{\prime \prime N}, \\
22^{\circ} 47^{\prime} 17^{\prime \prime} \mathrm{W}\end{array}$ & 170523 & 1668.2 & 15331 & MF491544 & 95026 & 80394 & $\begin{array}{l}\text { Africonus } \\
\text { maioensis }\end{array}$ \\
\hline 0392 & $\begin{array}{l}\text { Africonus } \\
\text { irregularis }\end{array}$ & $\begin{array}{l}\text { Baía da Gata, Boa Vista, } \\
\text { Cabo Verde }\end{array}$ & $\begin{array}{l}16^{\circ} 11^{\prime} 50^{\prime \prime} \mathrm{N} \\
22^{\circ} 42^{\prime} 32^{\prime \prime} \mathrm{W}\end{array}$ & 252126 & 2454.3 & 15324 & MF491550 & 95032 & 80400 & $\begin{array}{l}\text { Africonus } \\
\text { crotchii }\end{array}$ \\
\hline 1084 & $\begin{array}{l}\text { Africonus } \\
\text { irregularis }\end{array}$ & $\begin{array}{l}\text { Morro de Areia, Boa } \\
\text { Vista, Cabo Verde }\end{array}$ & $\begin{array}{l}16^{\circ} 5^{\prime} 24^{\prime \prime N} \\
22^{\circ} 57^{\prime} 7 " \mathrm{~W}\end{array}$ & 125264 & 1225.1 & 15330 & MF491593 & 95078 & 79953 & $\begin{array}{l}\text { Africonus } \\
\text { crotchii }\end{array}$ \\
\hline 1128 & $\begin{array}{l}\text { Africonus } \\
\text { irregularis }\end{array}$ & $\begin{array}{l}\text { Estancinha, Ponta do Sol, } \\
\text { Boa Vista, Cabo Verde }\end{array}$ & $\begin{array}{l}16^{\circ} 13^{\prime} 12^{\prime \prime} \mathrm{N} \\
22^{\circ} 55^{\prime} 9^{\prime \prime} \mathrm{W}\end{array}$ & 469101 & 4597.1 & 15313 & MF491597 & 95082 & 79997 & $\begin{array}{l}\text { Africonus } \\
\text { crotchii }\end{array}$ \\
\hline 0225 & $\begin{array}{l}\text { Africonus } \\
\text { isabelarum }\end{array}$ & $\begin{array}{l}\text { Ponta do Pau Seco, } \\
\text { Maio, Cabo Verde }\end{array}$ & $\begin{array}{l}15^{\circ} 15^{\prime} 26^{\prime \prime N}, \\
23^{\circ} 13^{\prime} 16^{\prime \prime} \mathrm{W}\end{array}$ & 247567 & 2431.7 & 15244 & MF491538 & 95020 & 78850 & - \\
\hline 0085 & $\begin{array}{l}\text { Africonus } \\
\text { josephinae }\end{array}$ & $\begin{array}{l}\text { Lage Branca, Maio, Cabo } \\
\text { Verde }\end{array}$ & $\begin{array}{l}15^{\circ} 18^{\prime} 32^{\prime \prime} \mathrm{N} \\
23^{\circ} 8^{\prime} 17^{\prime \prime} \mathrm{W}\end{array}$ & 224495 & 2204.6 & 15239 & MF491525 & 95007 & 78710 & $\begin{array}{l}\text { Africonus sp. } \\
\text { nov. } 2\end{array}$ \\
\hline 0555 & $\begin{array}{l}\text { Africonus } \\
\text { josephinae }\end{array}$ & $\begin{array}{l}\text { Ilhéu de Sal Rei, Boa } \\
\text { Vista, Cabo Verde }\end{array}$ & $\begin{array}{l}16^{\circ} 9^{\prime} 50^{\prime \prime} \mathrm{N} \\
22^{\circ} 55^{\prime} 31 " \mathrm{~W}\end{array}$ & 169723 & 1611.8 & 15330 & MF491565 & 95048 & 80415 & - \\
\hline 0830 & $\begin{array}{l}\text { Africonus } \\
\text { longilineus }\end{array}$ & $\begin{array}{l}\text { Serra Negra, Sal, Cabo } \\
\text { Verde }\end{array}$ & $\begin{array}{l}16^{\circ} 38^{\prime} 17^{\prime \prime} \mathrm{N} \\
22^{\circ} 53^{\prime} 56^{\prime \prime} \mathrm{W}\end{array}$ & 148726 & 1453 & 15316 & MF491572 & 95056 & 79739 & - \\
\hline 0847 & $\begin{array}{l}\text { Africonus } \\
\text { longilineus }\end{array}$ & $\begin{array}{l}\text { Rabo de Junco, Sal, Cabo } \\
\text { Verde }\end{array}$ & $\begin{array}{l}16^{\circ} 41^{\prime} 44^{\prime \prime} \mathrm{N}, \\
22^{\circ} 58^{\prime} 35^{\prime \prime} \mathrm{W}\end{array}$ & 308057 & 3000.9 & 15333 & MF491574 & 95058 & 79756 & $\begin{array}{l}\text { Africonus } \\
\text { miruchae }\end{array}$ \\
\hline 0410 & Africonus luquei & $\begin{array}{l}\text { Praia Canto, Boa Vista, } \\
\text { Cabo Verde }\end{array}$ & $\begin{array}{l}16^{\circ} 11^{\prime} 10^{\prime \prime} \mathrm{N} \\
22^{\circ} 42^{\prime} 28^{\prime \prime} \mathrm{W}\end{array}$ & 83198 & 815.1 & 15244 & MF491552 & 95034 & 80402 & $\begin{array}{l}\text { Africonus } \\
\text { delanoyae }\end{array}$ \\
\hline 0064 & $\begin{array}{l}\text { Africonus } \\
\text { maioensis }\end{array}$ & $\begin{array}{l}\text { Porto Cais, Maio, Cabo } \\
\text { Verde }\end{array}$ & $\begin{array}{l}15^{\circ} 19^{\prime} 15^{\prime \prime} \mathrm{N} \\
23^{\circ} 11^{\prime} 10^{\prime \prime} \mathrm{W}\end{array}$ & 143797 & 1402.9 & 15327 & MF491523 & 95005 & 78689 & - \\
\hline 0510 & $\begin{array}{l}\text { Africonus } \\
\text { marckeppensi }\end{array}$ & $\begin{array}{l}\text { Ervatao Norte, Boa Vista, } \\
\text { Cabo Verde }\end{array}$ & $\begin{array}{l}16^{\circ} 12^{\prime} 3^{\prime \prime} \mathrm{N} \\
22^{\circ} 54^{\prime} 43^{\prime \prime} \mathrm{W}\end{array}$ & 254030 & 2480.3 & 15330 & MF491562 & 95044 & 80412 & $\begin{array}{l}\text { Africonus } \\
\text { josephinae }\end{array}$ \\
\hline 0102 & & & & 80254 & 783.1 & 15326 & MF491527 & 95009 & 78727 & \\
\hline
\end{tabular}


Table 1 Mitochondrial (mt) genomes analyzed in this study (Continued)

\begin{tabular}{|c|c|c|c|c|c|c|c|c|c|c|}
\hline & $\begin{array}{l}\text { Africonus } \\
\text { marcocastellazzii }\end{array}$ & $\begin{array}{l}\text { Lage Branca, Maio, Cabo } \\
\text { Verde }\end{array}$ & $\begin{array}{l}15^{\circ} 18^{\prime} 32^{\prime \prime N}, \\
23^{\circ} 8^{\prime} 17^{\prime \prime} \mathrm{W}\end{array}$ & & & & & & & $\begin{array}{l}\text { Africonus } \\
\text { maioensis }\end{array}$ \\
\hline 0870 & $\begin{array}{l}\text { Africonus } \\
\text { melissae }\end{array}$ & $\begin{array}{l}\text { Baía da Parda, Sal, Cabo } \\
\text { Verde }\end{array}$ & $\begin{array}{l}16^{\circ} 45^{\prime} 7 " \mathrm{~N} \\
22^{\circ} 53^{\prime} 56^{\prime \prime} \mathrm{W}\end{array}$ & 195531 & 1907.9 & 15328 & MF491577 & 95061 & 79779 & $\begin{array}{l}\text { Africonus } \\
\text { longilineus }\end{array}$ \\
\hline 0455 & Africonus messiasi & $\begin{array}{l}\text { Derrubado (bay East), } \\
\text { Boa Vista, Cabo Verde }\end{array}$ & $\begin{array}{l}16^{\circ} 13^{\prime} 33^{\prime \prime} \mathrm{N}, \\
22^{\circ} 47^{\prime} 3^{\prime \prime} \mathrm{W}\end{array}$ & 250171 & 2447.7 & 15260 & MF491558 & 95040 & 80408 & $\begin{array}{l}\text { Africonus } \\
\text { fuscoflavus }\end{array}$ \\
\hline 0426 & $\begin{array}{l}\text { Africonus } \\
\text { miguelfiaderoi }\end{array}$ & $\begin{array}{l}\text { Praia Canto, Boa Vista, } \\
\text { Cabo Verde }\end{array}$ & $\begin{array}{l}16^{\circ} 11^{\prime} 10^{\prime \prime} \mathrm{N}, \\
22^{\circ} 42^{\prime} 28^{\prime \prime} \mathrm{W}\end{array}$ & 130100 & 1270.5 & 15328 & MF491554 & 95036 & 80404 & $\begin{array}{l}\text { Africonus } \\
\text { vulcanus }\end{array}$ \\
\hline 0905 & $\begin{array}{l}\text { Africonus } \\
\text { mordeirae }\end{array}$ & $\begin{array}{l}\text { Baía do Roucamento, Sal, } \\
\text { Cabo Verde }\end{array}$ & $\begin{array}{l}16^{\circ} 41^{\prime} 20^{\prime \prime} \mathrm{N}, \\
22^{\circ} 56^{\prime} 24^{\prime \prime} \mathrm{W}\end{array}$ & 99337 & 971.4 & 15241 & MF491580 & 95065 & 79814 & $\begin{array}{l}\text { Africonus } \\
\text { cuneolus }\end{array}$ \\
\hline 1091 & $\begin{array}{l}\text { Africonus } \\
\text { morroensis }\end{array}$ & $\begin{array}{l}\text { Morro de Areia, Boa } \\
\text { Vista, Cabo Verde }\end{array}$ & $\begin{array}{l}16^{\circ} 5^{\prime} 24^{\prime \prime N} \\
22^{\circ} 57^{\prime} 7 " \mathrm{~W}\end{array}$ & 172039 & 1515.1 & 15337 & MF491594 & 95079 & 79960 & $\begin{array}{l}\text { Africonus } \\
\text { diminutus }\end{array}$ \\
\hline 1395 & $\begin{array}{l}\text { Africonus } \\
\text { navarroi }\end{array}$ & $\begin{array}{l}\text { Calhau, São Vicente, } \\
\text { Cabo Verde }\end{array}$ & $\begin{array}{l}16^{\circ} 51^{\prime} 7 " \mathrm{~N} \\
24^{\circ} 51^{\prime} 59^{\prime \prime} \mathrm{W}\end{array}$ & 665250 & 6509.2 & 15331 & MF491600 & 95087 & 78557 & - \\
\hline 0250 & $\begin{array}{l}\text { Africonus } \\
\text { nelsontiagoi }\end{array}$ & $\begin{array}{l}\text { Tarrafal, Santiago, Cabo } \\
\text { Verde }\end{array}$ & $\begin{array}{l}15^{\circ} 16^{\prime} 50^{\prime \prime} \mathrm{N}, \\
23^{\circ} 45^{\prime} 15^{\prime \prime} \mathrm{W}\end{array}$ & 173873 & 1687.2 & 15339 & MF491541 & 95023 & 78875 & $\begin{array}{l}\text { Africonus } \\
\text { verdensis }\end{array}$ \\
\hline 0820 & $\begin{array}{l}\text { Africonus } \\
\text { pseudocuneolus }\end{array}$ & $\begin{array}{l}\text { Serra Negra, Sal, Cabo } \\
\text { Verde }\end{array}$ & $\begin{array}{l}16^{\circ} 38^{\prime} 17^{\prime \prime N}, \\
22^{\circ} 53^{\prime} 56^{\prime \prime} \mathrm{W}\end{array}$ & 131838 & 1288.7 & 15337 & MF491571 & 95055 & 79729 & $\begin{array}{l}\text { Africonus } \\
\text { cuneolus }\end{array}$ \\
\hline 0036 & $\begin{array}{l}\text { Africonus } \\
\text { raulsilvai }\end{array}$ & $\begin{array}{l}\text { Praia da Soca, Maio, } \\
\text { Cabo Verde }\end{array}$ & $\begin{array}{l}15^{\circ} 15^{\prime} 8^{\prime \prime N} \\
23^{\circ} 13^{\prime} 4^{\prime \prime} \mathrm{W}\end{array}$ & 345872 & 1699.6 & 15534 & MF491520 & 95002 & 78661 & - \\
\hline 0865 & Africonus regonae & $\begin{array}{l}\text { Baía da Fontona, Sal, } \\
\text { Cabo Verde }\end{array}$ & $\begin{array}{l}16^{\circ} 44^{\prime} 22^{\prime \prime} \mathrm{N}, \\
22^{\circ} 58^{\prime} 466^{\prime \prime} \mathrm{W}\end{array}$ & 246041 & 2411 & 15328 & MF491576 & 95060 & 79774 & - \\
\hline 0950 & Africonus regonae & Regona, Sal, Cabo Verde & $\begin{array}{l}16^{\circ} 48^{\prime} 5^{\prime \prime} \mathrm{N}_{1} \\
22^{\circ} 59^{\prime} 33^{\prime \prime} \mathrm{W}\end{array}$ & 88673 & 864.1 & 15337 & MF491585 & 95070 & 79858 & - \\
\hline 0586 & Africonus roeckeli & $\begin{array}{l}\text { Praia Canto, Boa Vista, } \\
\text { Cabo Verde }\end{array}$ & $\begin{array}{l}16^{\circ} 11^{\prime} 10^{\prime \prime} \mathrm{N}, \\
22^{\circ} 42^{\prime} 28^{\prime \prime} \mathrm{W}\end{array}$ & 141600 & 1385.7 & 15320 & MF491567 & 95050 & 80417 & - \\
\hline 0549 & $\begin{array}{l}\text { Africonus } \\
\text { salreiensis }\end{array}$ & $\begin{array}{l}\text { Ilhéu de Sal Rei, Boa } \\
\text { Vista, Cabo Verde }\end{array}$ & $\begin{array}{l}16^{\circ} 9^{\prime} 50^{\prime \prime} \mathrm{N} \\
22^{\circ} 55^{\prime} 31 \text { "W }\end{array}$ & 349070 & 3402.9 & 15331 & MF491564 & 95047 & 80414 & $\begin{array}{l}\text { Africonus } \\
\text { crotchii }\end{array}$ \\
\hline 0810 & $\begin{array}{l}\text { Africonus } \\
\text { serranegrae }\end{array}$ & $\begin{array}{l}\text { Serra Negra, Sal, Cabo } \\
\text { Verde }\end{array}$ & $\begin{array}{l}16^{\circ} 38^{\prime} 17^{\prime \prime} \mathrm{N}, \\
22^{\circ} 53^{\prime} 56^{\prime \prime} \mathrm{W}\end{array}$ & 182124 & 1777.6 & 15335 & MF491570 & 95054 & 79719 & $\begin{array}{l}\text { Africonus } \\
\text { cuneolus }\end{array}$ \\
\hline 1078 & Africonus silviae & $\begin{array}{l}\text { Morro de Areia, Boa } \\
\text { Vista, Cabo Verde }\end{array}$ & $\begin{array}{l}16^{\circ} 5^{\prime} 24^{\prime \prime N}, \\
22^{\circ} 57^{\prime} 7 " \mathrm{~W}\end{array}$ & 293568 & 2876.1 & 15336 & MF491592 & 95077 & 79947 & $\begin{array}{l}\text { Africonus } \\
\text { fuscoflavus }\end{array}$ \\
\hline 0445 & $\begin{array}{l}\text { Africonus } \\
\text { swinneni }\end{array}$ & $\begin{array}{l}\text { Porto Ferreira, Boa Vista, } \\
\text { Cabo Verde }\end{array}$ & $\begin{array}{l}16^{\circ} 7^{\prime} 45^{\prime \prime} \mathrm{N} \\
22^{\circ} 40^{\prime} 17^{\prime \prime} \mathrm{W}\end{array}$ & 221672 & 2169.1 & 15244 & MF491556 & 95038 & 80406 & $\begin{array}{l}\text { Africonus } \\
\text { delanoyae }\end{array}$ \\
\hline 1125 & $\begin{array}{l}\text { Africonus } \\
\text { teodorae }\end{array}$ & $\begin{array}{l}\text { Estancinha, Ponta do Sol, } \\
\text { Boa Vista, Cabo Verde }\end{array}$ & $\begin{array}{l}16^{\circ} 13^{\prime} 12^{\prime \prime} \mathrm{N}, \\
22^{\circ} 55^{\prime} 9^{\prime \prime} \mathrm{W}\end{array}$ & 326654 & 3185.5 & 15334 & MF491596 & 95081 & 79994 & $\begin{array}{l}\text { Africonus } \\
\text { crotchii }\end{array}$ \\
\hline 1035 & $\begin{array}{l}\text { Africonus } \\
\text { umbelinae }\end{array}$ & $\begin{array}{l}\text { Espingueira, Boa Vista, } \\
\text { Cabo Verde }\end{array}$ & $\begin{array}{l}16^{\circ} 12^{\prime} 55^{\prime \prime} \mathrm{N} \\
22^{\circ} 47^{\prime} 49^{\prime \prime} \mathrm{W}\end{array}$ & 110526 & 1085.5 & 15333 & MF491589 & 95074 & 79904 & $\begin{array}{l}\text { Africonus } \\
\text { damottai }\end{array}$ \\
\hline 0240 & $\begin{array}{l}\text { Africonus } \\
\text { verdensis }\end{array}$ & $\begin{array}{l}\text { Tarrafal, Santiago, Cabo } \\
\text { Verde }\end{array}$ & $\begin{array}{l}15^{\circ} 16^{\prime} 50^{\prime \prime} \mathrm{N}, \\
23^{\circ} 45^{\prime} 15^{\prime \prime} \mathrm{W}\end{array}$ & 53400 & 519.8 & 15339 & MF491540 & 95022 & 78865 & - \\
\hline 0435 & $\begin{array}{l}\text { Africonus } \\
\text { vulcanus }\end{array}$ & $\begin{array}{l}\text { Porto Ferreira, Boa Vista, } \\
\text { Cabo Verde }\end{array}$ & $\begin{array}{l}16^{\circ} 7^{\prime} 45^{\prime \prime} \mathrm{N} \\
22^{\circ} 40^{\prime} 17^{\prime \prime} \mathrm{W}\end{array}$ & 284766 & 2787.4 & 15242 & MF491555 & 95037 & 80405 & - \\
\hline 1110 & Africonus zinhoi & $\begin{array}{l}\text { Curral Velho, Boa Vista, } \\
\text { Cabo Verde }\end{array}$ & $\begin{array}{l}15^{\circ} 58^{\prime} 4^{\prime \prime} N_{1} \\
22^{\circ} 47^{\prime} 42^{\prime \prime} W\end{array}$ & 388828 & 3785.5 & 15331 & MF491595 & 95080 & 79979 & $\begin{array}{l}\text { Africonus } \\
\text { maioensis }\end{array}$ \\
\hline 7036 & $\begin{array}{l}\text { Trovaoconus } \\
\text { atlanticoselvagem }\end{array}$ & $\begin{array}{l}\text { Baixo João Valente, Cabo } \\
\text { Verde }\end{array}$ & $\begin{array}{l}15^{\circ} 44^{\prime} 27^{\prime \prime} \mathrm{N} \\
23^{\circ} 5^{\prime} 26^{\prime \prime} \mathrm{W}\end{array}$ & 95264 & 933.8 & 15352 & MF491606 & 7036 & - & $\begin{array}{l}\text { Kalloconus } \\
\text { trochulus }\end{array}$ \\
\hline 0616 & $\begin{array}{l}\text { Trovaoconus cf. } \\
\text { ateralbus }\end{array}$ & $\begin{array}{l}\text { Serra Negra, Sal, Cabo } \\
\text { Verde }\end{array}$ & $\begin{array}{l}16^{\circ} 38^{\prime} 17^{\prime \prime N}, \\
22^{\circ} 53^{\prime} 56^{\prime \prime} \mathrm{W}\end{array}$ & 87550 & 853.7 & 15344 & MF491568 & 95052 & 79664 & $\begin{array}{l}\text { Kalloconus } \\
\text { sp. nov. } 1\end{array}$ \\
\hline 0010 & $\begin{array}{l}\text { Trovaoconus } \\
\text { pseudonivifer }\end{array}$ & $\begin{array}{l}\text { Ponta do Pau Seco, } \\
\text { Maio, Cabo Verde }\end{array}$ & $\begin{array}{l}15^{\circ} 15^{\prime} 26^{\prime \prime} \mathrm{N}, \\
23^{\circ} 13^{\prime} 16^{\prime \prime} \mathrm{W}\end{array}$ & 56486 & 555.5 & 15351 & MF491519 & 95000 & 78635 & $\begin{array}{l}\text { Kalloconus } \\
\text { trochulus }\end{array}$ \\
\hline 0094 & $\begin{array}{l}\text { Trovaoconus } \\
\text { pseudonivifer }\end{array}$ & $\begin{array}{l}\text { Lage Branca, Maio, Cabo } \\
\text { Verde }\end{array}$ & $\begin{array}{l}15^{\circ} 18^{\prime} 32^{\prime \prime} \mathrm{N}, \\
23^{\circ} 8^{\prime} 177^{\prime \prime}\end{array}$ & 454415 & 4429.8 & 15351 & MF491526 & 95008 & 78719 & $\begin{array}{l}\text { Kalloconus } \\
\text { trochulus }\end{array}$ \\
\hline 0154 & $\begin{array}{l}\text { Trovaoconus } \\
\text { pseudonivifer }\end{array}$ & $\begin{array}{l}\text { Porto Cais (north), Maio, } \\
\text { Cabo Verde }\end{array}$ & $\begin{array}{l}15^{\circ} 19^{\prime} 45^{\prime \prime} \mathrm{N} \\
23^{\circ} 10^{\prime} 57^{\prime \prime} \mathrm{W}\end{array}$ & 199736 & 1954.2 & 15352 & MF491532 & 95014 & 78779 & $\begin{array}{l}\text { Kalloconus } \\
\text { trochulus }\end{array}$ \\
\hline 0420 & $\begin{array}{l}\text { Trovaoconus } \\
\text { pseudonivifer }\end{array}$ & $\begin{array}{l}\text { Praia Canto, Boa Vista, } \\
\text { Cabo Verde }\end{array}$ & $\begin{array}{l}16^{\circ} 11^{\prime} 10^{\prime \prime} \mathrm{N}, \\
22^{\circ} 42^{\prime} 28^{\prime \prime} \mathrm{W}\end{array}$ & 111182 & 1085 & 15347 & MF491553 & 95035 & 80403 & $\begin{array}{l}\text { Kalloconus } \\
\text { pseudonivifer }\end{array}$ \\
\hline 0500 & & & & 223915 & 2177.2 & 15351 & MF491561 & 95043 & 80411 & \\
\hline
\end{tabular}


Table 1 Mitochondrial (mt) genomes analyzed in this study (Continued)

\begin{tabular}{|c|c|c|c|c|c|c|c|c|c|c|}
\hline & $\begin{array}{l}\text { Trovaoconus } \\
\text { trochulus }\end{array}$ & $\begin{array}{l}\text { Baía do Ervatão (North), } \\
\text { Boa Vista, Cabo Verde }\end{array}$ & $\begin{array}{l}16^{\circ} 12^{\prime} 3 " \mathrm{~N} \\
22^{\circ} 54^{\prime} 43^{\prime \prime} \mathrm{W}\end{array}$ & & & & & & & $\begin{array}{l}\text { Kalloconus } \\
\text { trochulus }\end{array}$ \\
\hline 0149 & $\begin{array}{l}\text { Trovaoconus } \\
\text { venulatus }\end{array}$ & $\begin{array}{l}\text { Lage Branca, Maio, Cabo } \\
\text { Verde }\end{array}$ & $\begin{array}{l}15^{\circ} 18^{\prime} 32^{\prime \prime} \mathrm{N} \\
23^{\circ} 8^{\prime} 17^{\prime \prime} \mathrm{W}\end{array}$ & 105732 & 1014.9 & 15276 & MF491531 & 95013 & 78774 & $\begin{array}{l}\text { Kalloconus } \\
\text { venulatus }\end{array}$ \\
\hline 0187 & $\begin{array}{l}\text { Trovaoconus } \\
\text { venulatus }\end{array}$ & $\begin{array}{l}\text { Praia Real, Maio, Cabo } \\
\text { Verde }\end{array}$ & $\begin{array}{l}15^{\circ} 19^{\prime} 45^{\prime \prime} \mathrm{N} \\
23^{\circ} 10^{\prime} 40^{\prime \prime} \mathrm{W}\end{array}$ & 144651 & 1415 & 15330 & MF491535 & 95017 & 78812 & $\begin{array}{l}\text { Kalloconus } \\
\text { venulatus }\end{array}$ \\
\hline 0234 & $\begin{array}{l}\text { Trovaoconus } \\
\text { venulatus }\end{array}$ & $\begin{array}{l}\text { Ponta do Pau Seco, } \\
\text { Maio, Cabo Verde }\end{array}$ & $\begin{array}{l}15^{\circ} 15^{\prime} 26^{\prime \prime} \mathrm{N} \\
23^{\circ} 13^{\prime} 17^{\prime \prime} \mathrm{W}\end{array}$ & 67867 & 661.5 & 15320 & MF491539 & 95021 & 78859 & $\begin{array}{l}\text { Kalloconus } \\
\text { venulatus }\end{array}$ \\
\hline 0347 & $\begin{array}{l}\text { Trovaoconus } \\
\text { venulatus }\end{array}$ & $\begin{array}{l}\text { Derrubado (bay West), } \\
\text { Boa Vista, Cabo Verde }\end{array}$ & $\begin{array}{l}16^{\circ} 13^{\prime} 22^{\prime \prime} \mathrm{N} \\
22^{\circ} 47^{\prime} 41^{\prime \prime} \mathrm{W}\end{array}$ & 48636 & 475.9 & 15326 & MF491546 & 95028 & 80396 & $\begin{array}{l}\text { Kalloconus } \\
\text { venulatus }\end{array}$ \\
\hline 0475 & $\begin{array}{l}\text { Trovaoconus } \\
\text { venulatus }\end{array}$ & $\begin{array}{l}\text { Ponta Antónia, Boa Vista, } \\
\text { Cabo Verde }\end{array}$ & $\begin{array}{l}16^{\circ} 13^{\prime} 24^{\prime \prime} \mathrm{N} \\
22^{\circ} 46^{\prime} 59^{\prime \prime} \mathrm{W}\end{array}$ & 409041 & 3966.1 & 15340 & MF491560 & 95042 & 80410 & $\begin{array}{l}\text { Kalloconus } \\
\text { venulatus }\end{array}$ \\
\hline 1038 & $\begin{array}{l}\text { Trovaoconus } \\
\text { venulatus }\end{array}$ & $\begin{array}{l}\text { Praia Canto, Boa Vista, } \\
\text { Cabo Verde }\end{array}$ & $\begin{array}{l}16^{\circ} 11^{\prime} 10^{\prime \prime} \mathrm{N} \\
22^{\circ} 42^{\prime} 28^{\prime \prime} \mathrm{W}\end{array}$ & 143644 & 2403.8 & 15336 & MF491590 & 95075 & 79907 & $\begin{array}{l}\text { Kalloconus } \\
\text { venulatus }\end{array}$ \\
\hline |B001 & $\begin{array}{l}\text { Lautoconus } \\
\text { ventricosus }\end{array}$ & $\begin{array}{l}\text { Estani des Peix, } \\
\text { Formentera, Balearic } \\
\text { Islands, Spain }\end{array}$ & $\begin{array}{l}38^{\circ} 43^{\prime} 49^{\prime \prime} \mathrm{N} \\
1^{\circ} 24^{\prime} 42^{\prime \prime} \mathrm{E}\end{array}$ & 86290 & 842.5 & 15341 & MF491607 & 95094 & 80426 & $\begin{array}{l}\text { Lautoconus } \\
\text { sp. nov } 1\end{array}$ \\
\hline \multicolumn{11}{|c|}{ GenBank mt genomes } \\
\hline ID & Species & Location & Coordinates & \multicolumn{2}{|c|}{ Reference } & $\begin{array}{l}\text { Length } \\
\text { (bp) }\end{array}$ & $\begin{array}{l}\text { GenBank } \\
\text { Acc. No }\end{array}$ & $\begin{array}{l}\text { Voucher } \\
\text { (MNCN/ADN) }\end{array}$ & $\begin{array}{l}\text { Voucher shell } \\
\text { (MNCN 15.05/) }\end{array}$ & $\begin{array}{l}\text { New species } \\
\text { proposed }^{\mathrm{a}}\end{array}$ \\
\hline 6990 & Africonus borgesi & $\begin{array}{l}\text { Porto Ferreira, Boa Vista, } \\
\text { Cabo Verde }\end{array}$ & $\begin{array}{l}16^{\circ} 7^{\prime} 45^{\prime \prime} \mathrm{N} \\
22^{\circ} \\
40^{\prime} 170^{\prime \prime} \mathrm{W}\end{array}$ & \multicolumn{2}{|c|}{$\begin{array}{l}\text { Cunha et al., } \\
\text { (2009) }\end{array}$} & 15536 & NC_013243 & 6990 & - & - \\
\hline 0025 & Africonus infinitus & $\begin{array}{l}\text { Ponta do Pau Seco, } \\
\text { Maio, Cabo Verde }\end{array}$ & $\begin{array}{l}15^{\circ} 15^{\prime} 26^{\prime \prime} \mathrm{N} \\
23^{\circ} 13^{\prime} 17^{\prime \prime} \mathrm{W}\end{array}$ & \multicolumn{2}{|c|}{$\begin{array}{l}\text { Abalde et al., } \\
\text { (in prep.) }\end{array}$} & 15522 & KY864967 & 95001 & 78650 & - \\
\hline 0875 & $\begin{array}{l}\text { Africonus } \\
\text { miruchae }\end{array}$ & $\begin{array}{l}\text { Terrinha Fina, Palhona, } \\
\text { Sal, Cabo Verde }\end{array}$ & $\begin{array}{l}16^{\circ} 49^{\prime} 12^{\prime \prime} \mathrm{N} \\
22^{\circ} 59^{\prime} 12^{\prime \prime} \mathrm{W}\end{array}$ & \multicolumn{2}{|c|}{$\begin{array}{l}\text { Abalde et al., } \\
\text { (in prep.) }\end{array}$} & 15336 & KY864971 & 95062 & 79784 & - \\
\hline 0534 & $\begin{array}{l}\text { Trovaoconus } \\
\text { pseudonivifer }\end{array}$ & $\begin{array}{l}\text { Estancinha, Ponta do Sol, } \\
\text { Boa Vista, Cabo Verde }\end{array}$ & $\begin{array}{l}16^{\circ} 13^{\prime} 12^{\prime \prime} \mathrm{N} \\
22^{\circ} 55^{\prime} 9^{\prime \prime} \mathrm{W}\end{array}$ & \multicolumn{2}{|c|}{$\begin{array}{l}\text { Abalde et al., } \\
\text { (in prep.) }\end{array}$} & 15351 & KY864969 & 95046 & 80418 & $\begin{array}{l}\text { Kalloconus } \\
\text { trochulus }\end{array}$ \\
\hline 0550 & $\begin{array}{l}\text { Trovaoconus } \\
\text { venulatus }\end{array}$ & $\begin{array}{l}\text { Ilhéu de Sal Rei, Boa } \\
\text { Vista, Cabo Verde }\end{array}$ & $\begin{array}{l}16^{\circ} 9^{\prime} 56^{\prime \prime} \mathrm{N} \\
22^{\circ} 55^{\prime} 23^{\prime \prime} \mathrm{W}\end{array}$ & \multicolumn{2}{|c|}{$\begin{array}{l}\text { Uribe et al., } \\
\text { (2017) }\end{array}$} & 15524 & KX263250 & 86741 & 80419 & $\begin{array}{l}\text { Kalloconus } \\
\text { venulatus }\end{array}$ \\
\hline 0601 & $\begin{array}{l}\text { Trovaoconus } \\
\text { ateralbus }\end{array}$ & $\begin{array}{l}\text { Calheta Funda, Sal, Cabo } \\
\text { Verde }\end{array}$ & $\begin{array}{l}16^{\circ} 39^{\prime} 6^{\prime \prime} \mathrm{N} \\
22^{\circ} 56^{\prime} 53^{\prime \prime} \mathrm{W}\end{array}$ & \multicolumn{2}{|c|}{$\begin{array}{l}\text { Abalde et al., } \\
\text { (in prep.) }\end{array}$} & 15327 & KY864970 & 95051 & 79649 & $\begin{array}{l}\text { Kalloconus } \\
\text { ateralbus }\end{array}$ \\
\hline 1375 & $\begin{array}{l}\text { Kalloconus of. } \\
\text { byssinus }\end{array}$ & North Senegal & unknown & \multicolumn{2}{|c|}{$\begin{array}{l}\text { Abalde et al., } \\
\text { (in prep.) }\end{array}$} & 15348 & KY864973 & 95085 & 78536 & $\begin{array}{l}\text { Kalloconus } \\
\text { pulcher }\end{array}$ \\
\hline 1253 & $\begin{array}{l}\text { Kalloconus } \\
\text { pulcher }\end{array}$ & $\begin{array}{l}\text { Les Almadies, Dakar, } \\
\text { Senegal }\end{array}$ & $\begin{array}{l}14^{\circ} 44^{\prime} 40^{\prime \prime} \mathrm{N} \\
17^{\circ} \\
31^{\prime} 442^{\prime \prime} \mathrm{W}\end{array}$ & \multicolumn{2}{|c|}{$\begin{array}{l}\text { Abalde et al., } \\
\text { (in prep.) }\end{array}$} & 15332 & KY864972 & 95084 & 78414 & $\begin{array}{l}\text { Kalloconus } \\
\text { pulcher }\end{array}$ \\
\hline 1343 & $\begin{array}{l}\text { Lautoconus } \\
\text { belairensis }\end{array}$ & Terrou-Bi. Dakar, Senegal & $\begin{array}{l}14^{\circ} 40^{\prime} 29^{\prime \prime} \mathrm{N} \\
17^{\circ} 28^{\prime} 12^{\prime \prime} \mathrm{W}\end{array}$ & \multicolumn{2}{|c|}{$\begin{array}{l}\text { Abalde et al., } \\
\text { (2017) }\end{array}$} & 15321 & KY801849 & 91293 & 78504 & $\begin{array}{l}\text { Gen. nov. } \\
\text { belairensis }\end{array}$ \\
\hline 1338 & $\begin{array}{l}\text { Lautoconus } \\
\text { bruguieresi }\end{array}$ & $\begin{array}{l}\text { Île de Gorée, Dakar, } \\
\text { Senegal }\end{array}$ & $\begin{array}{l}14^{\circ} 40^{\prime} 16^{\prime \prime} \mathrm{N} \\
17^{\circ} 23^{\prime} 58^{\prime \prime} \mathrm{W}\end{array}$ & \multicolumn{2}{|c|}{$\begin{array}{l}\text { Abalde et al., } \\
\text { (2017) }\end{array}$} & 15340 & KY801851 & 91291 & 78499 & $\begin{array}{l}\text { Gen. nov. } \\
\text { bruguieresi }\end{array}$ \\
\hline 1296 & $\begin{array}{l}\text { Lautoconus } \\
\text { cloveri }\end{array}$ & Ndayane, Senegal & $\begin{array}{l}14^{\circ} 33^{\prime} 45^{\prime \prime} \mathrm{N} \\
17^{\circ} 7^{\prime} 34^{\prime \prime} \mathrm{W}\end{array}$ & \multicolumn{2}{|c|}{$\begin{array}{l}\text { Abalde et al., } \\
\text { (2017) }\end{array}$} & 15323 & KY801859 & 91283 & 78457 & $\begin{array}{l}\text { Gen. nov. } \\
\text { cloveri }\end{array}$ \\
\hline CG13 & $\begin{array}{l}\text { Lautoconus } \\
\text { guanche }\end{array}$ & $\begin{array}{l}\text { Lanzarote, Canary Islands, } \\
\text { Spain }\end{array}$ & $\begin{array}{l}28^{\circ} 57^{\prime} 16^{\prime \prime} \mathrm{N} \\
13^{\circ} 34^{\prime} 22^{\prime \prime} \mathrm{W}\end{array}$ & \multicolumn{2}{|c|}{$\begin{array}{l}\text { Abalde et al., } \\
(2017)\end{array}$} & 15506 & KY801847 & 91295 & - & $\begin{array}{l}\text { Gen. nov. } \\
\text { guanche }\end{array}$ \\
\hline 1266 & $\begin{array}{l}\text { Lautoconus } \\
\text { hybridus }\end{array}$ & NGor, Dakar, Senegal & $\begin{array}{l}14^{\circ} 45^{\prime} 67^{\prime \prime N} \\
17^{\circ} \\
30^{\prime} 36.33^{\prime \prime} \mathrm{W}\end{array}$ & \multicolumn{2}{|c|}{$\begin{array}{l}\text { Abalde et al., } \\
(2017)\end{array}$} & 15507 & KY801863 & 91279 & 78427 & $\begin{array}{l}\text { Gen. nov. } \\
\text { hybridus }\end{array}$ \\
\hline 1278 & $\begin{array}{l}\text { Lautoconus } \\
\text { mercator }\end{array}$ & NGor, Dakar, Senegal & $\begin{array}{l}14^{\circ} 45^{\prime} 6^{\prime \prime} \mathrm{N} \\
17^{\circ} 30^{\prime} 36^{\prime \prime} \mathrm{W}\end{array}$ & \multicolumn{2}{|c|}{$\begin{array}{l}\text { Abalde et al., } \\
\text { (2017) }\end{array}$} & 15329 & KY801862 & 91280 & 78439 & $\begin{array}{l}\text { Gen. nov. } \\
\text { mercator }\end{array}$ \\
\hline CV13 & $\begin{array}{l}\text { Lautoconus } \\
\text { ventricosus }\end{array}$ & $\begin{array}{l}\text { Ria Formosa, Faro, } \\
\text { Portugal }\end{array}$ & $\begin{array}{l}36^{\circ} 58^{\prime} 0^{\prime \prime N}, \\
7^{\circ} 53^{\prime} 2 " \mathrm{~W}\end{array}$ & \multicolumn{2}{|c|}{$\begin{array}{l}\text { Uribe et al., } \\
\text { (2017) }\end{array}$} & 15534 & KX263251 & 86742 & - & - \\
\hline CVERM1 & $\begin{array}{l}\text { Chelyconus } \\
\text { ermineus }\end{array}$ & $\begin{array}{l}\text { Praia Gonçalo, Maio, } \\
\text { Cabo Verde }\end{array}$ & $\begin{array}{l}15^{\circ} 16^{\prime} 13^{\prime \prime} \mathrm{N} \\
23^{\circ} 6^{\prime} 15^{\prime \prime} \mathrm{W}\end{array}$ & \multicolumn{2}{|c|}{$\begin{array}{l}\text { Abalde et al., } \\
\text { (in prep.) }\end{array}$} & 15365 & KY864977 & 95095 & 78876 & - \\
\hline
\end{tabular}




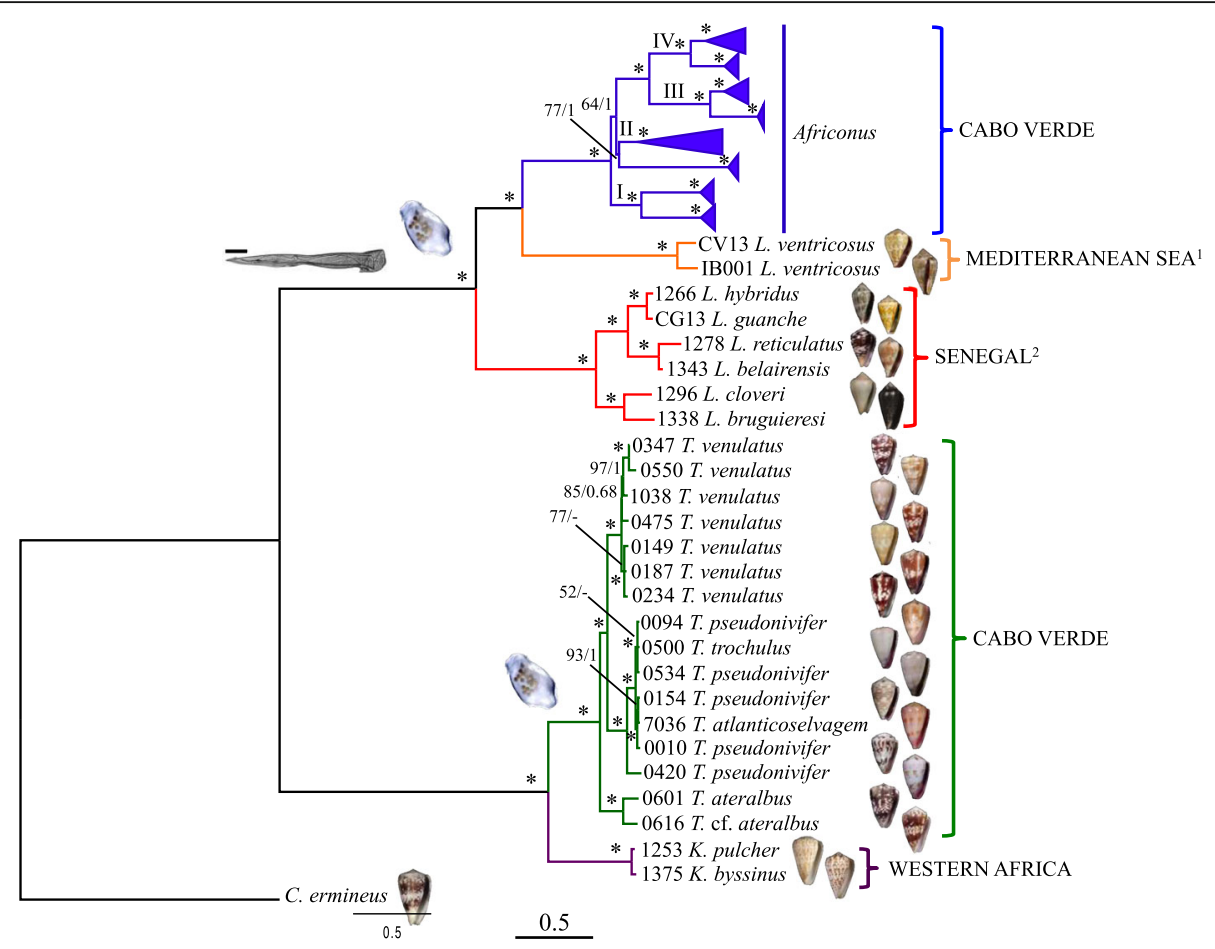

Fig. 2 Closest sister groups of cone snails endemic to Cabo Verde based on mt genomes (concatenated protein coding plus rRNA genes analyzed at the nucleotide level). The reconstructed ML tree using C. ermineus as outgroup is shown. Number of specimen, initial species assignment, and a ventral picture of the shell are provided. Numbers at nodes are statistical support values for ML (bootstrap proportions)/Bl (posterior probabilities). Scale bar indicates substitutions/site. Two major clades are recovered: the first one includes Kalloconus from Western Africa (purple) and Trovaoconus from Cabo Verde (green) whereas the second one includes Lautoconus from the Mediterranean Sea (and neighboring Atlantic Ocean'; orange), Lautoconus from Senegal (and L. guanche from Canary Islands'; red), and Africonus (blue) from Cabo Verde. Lineages within Africonus are expanded in Fig. 3. The "robust" type of radular tooth is shown as the ancestral character state for the second clade (scale bar equals $0.1 \mathrm{~mm}$; see diversity of radular teeth of the first clade in Additional file 1). The two transitions from planktonic to non-planktonic larvae are indicated by a sac of eggs

into two monophyletic groups (Figs. 2 and 3). Lineage I was the sister group of the remaining Africonus and its two lineages had each species from Maio sister to species from Boa Vista (Fig. 3a). Lineage II included species from Santiago and Maio sister to species endemic to the westernmost islands (Santo Antão, São Vicente and Santa Luzia). These latter species could be grouped into three main lineages, one containing species endemic to São Vicente, another containing species distributed both in Santa Luzia and São Vicente, and the third one including species from the three islands (Fig. 3a). Lineage III included species from Maio sister to species from Boa Vista (Fig. 3a). Lineage IV contained specimens representing most of the described species of Africonus. One monophyletic group included species endemic to Sal whereas the other clade included Africonus isabelarum from Maio as sister to four lineages, two containing exclusively species from Boa Vista, one having species from Maio sister to Africonus irregularis from Boa Vista, and one having species from Boa Vista and Africonus fantasmalis from Maio (Fig. 3b).
Pairwise uncorrected sequence divergences were estimated based on the alignment including the nucleotide sequences of the $13 \mathrm{mt}$ protein-coding and two rRNA genes. Pairwise uncorrected sequence divergences between C. ermineus and ingroup taxa averaged 18\%. The average pairwise uncorrected sequence divergence between the two main ingroup clades (genera Kalloconus + Trovaoconus versus genera Lautoconus + Africonus) was $16 \%$. Pairwise uncorrected sequence divergences between Lautoconus endemic to Senegal (plus L. guanche) and $L$. ventricosus plus Africonus averaged $11 \%$. The average pairwise uncorrected sequence divergence between the sister groups L. ventricosus and Africonus was 10\% whereas between Kalloconus and Trovaoconus, it was $5 \%$. The pairwise uncorrected sequence divergences between the four main lineages within Africonus averaged $6 \%$. The corresponding values for the pairwise divergences between the two major clades defined within each of the lineages I-IV were $4 \%, 6 \%, 3 \%$, and $3 \%$, respectively. Pairwise uncorrected sequence divergence comparisons between sister species level were distributed into two different ranges, one closer to $1 \%$ (0.5- 

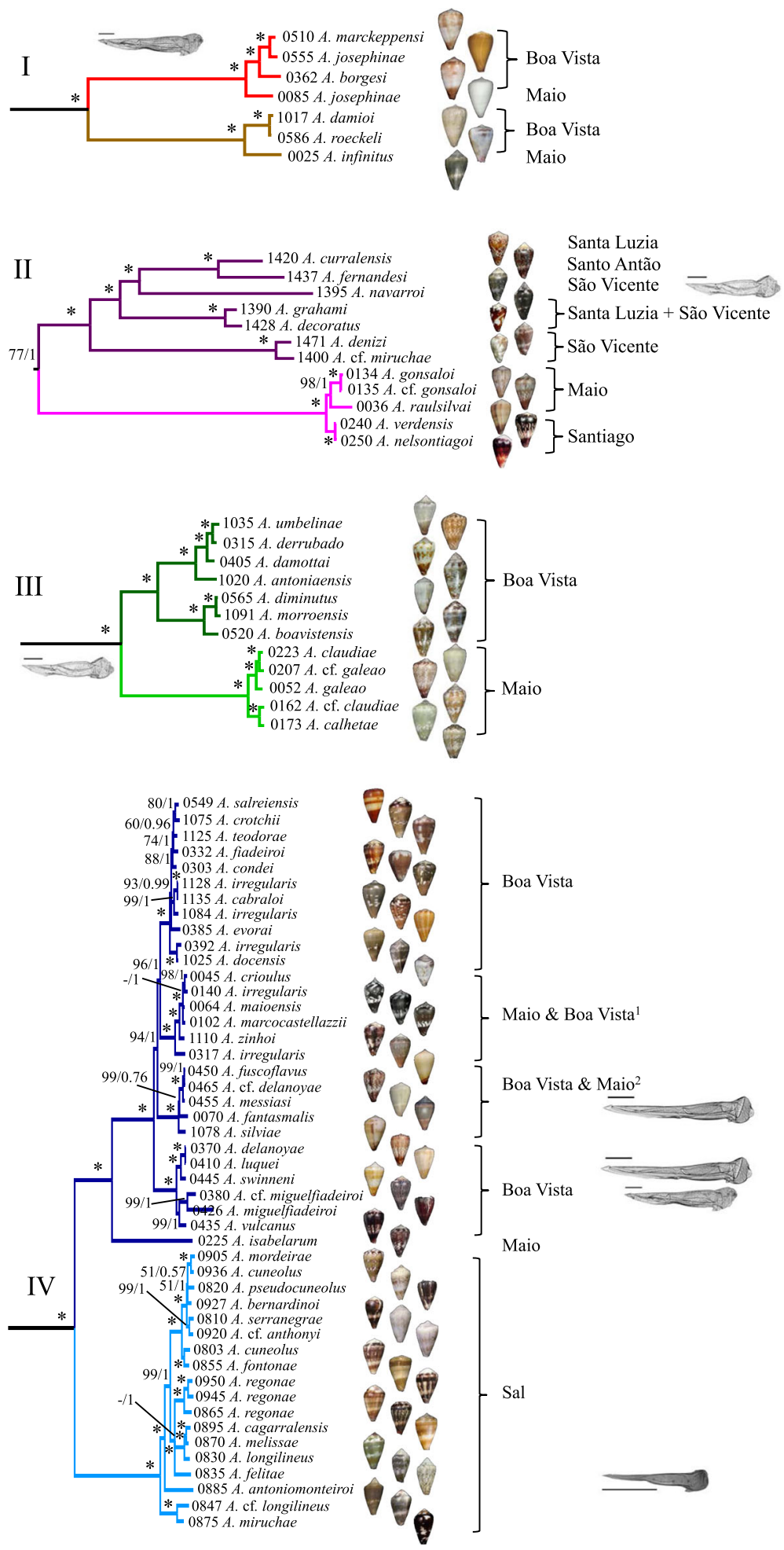

0.3

Fig. 3 (See legend on next page.) 
(See figure on previous page.)

Fig. 3 Phylogeny of Africonus based on mt genomes (concatenated protein coding plus rRNA genes analyzed at the nucleotide level). Number of specimen, initial species assignment, island, and a ventral picture of the shell are provided. Numbers at nodes are statistical support values for ML (bootstrap proportions)/BI (posterior probabilities). Hyphen indicates a bootstrap value below 50\%. Scale bar indicates substitutions/site. Four major lineages (I-IV) are recovered and indicated with different colors. All Africonus have the "robust" type of radular tooth except when indicated (scale bar equals $0.1 \mathrm{~mm}$ ). ${ }^{1}$ All taxa endemic to Maio except A. irregularis endemic to Boa Vista. ${ }^{2}$ All taxa endemic to Boa Vista except $A$.

fantamalis endemic to Maio

$1.5 \%)$ and the other closer to $0 \%(0-0.5 \%)$. The latter divergences were particularly abundant among sister species comparisons within Maio, Boa Vista and Sal. Several $\mathrm{mt}$ genomes of different species were almost identical $(<0.05 \%)$ in sequence including (1) Africonus delanoyae and Africonus luquei, (2) Africonus fuscoflavus, Africonus cf. delanoyae, and Africonus messiasi, (3) Africonus irregularis (\#1128) and Africonus cabraloi, (4) Africonus verdensis and Africonus nelsontiagoi, and (5) Africonus gonsaloi and Africonus cf. gonsaloi.

\section{Evolution of radular types}

The different lineages within Africonus exhibit distinct radular types (Fig. 3). Most lineages and species showed the "robust" type, which is of medium relative size, with a short, pointed barb and a basal spur (see Additional file 1). The anterior section of the tooth is equal or slightly shorter than the posterior section, and the blade covers most of the anterior section $(80 \%-85 \%)$. There are usually 19 to 30 denticles in the serration, arranged in one row (occasionally two). Several species within lineage IV (Africonus delanoyae, Africonus luquei, Africonus swinneni, Africonus fuscoflavus, Africonus messiasi, Africonus silviae and Africonus cf. delanoyae from Boa Vista island, and Africonus fantasmalis from Maio island) exhibited radular teeth of the "elongated" type, similar to the "robust" type but characterized by an anterior section which is longer than the posterior section, a blade covering 40 to $50 \%$ of the anterior section, and more numerous denticles in the serration (usually more than 30) often arranged in two rows. Several species (Africonus borgesi, Africonus josephinae and Africonus marckeppensi in lineage I, Africonus navarroi in lineage II), all species in lineage III, plus Africonus vulcanus, Africonus miguelfiadeiroi and Africonus cf. miguelfiadeiroi in lineage IV) displayed radular tooth of the "broad" type, which is characterized by a medium-sized (Shell Length/Tooth Length $=32-45$ ) and very broad radular tooth (Shell Length/Anterior section Width $=7-12$ ), with an anterior section which is shorter than the posterior section (Tooth Length/Anterior section Length $=2.1-2.9$ ), a blade covering most of the anterior section, and with a variable number of denticles (8 to 30) in the serration arranged in two or more rows. The radular morphology of Africonus felitae may represent a special case with a small relative size (Shell Length/Tooth Length $=63-67$ ), narrow (Tooth Length/Anterior section Width $=20-23$ ), the anterior section shorter than the posterior section (Tooth Length/Anterior section Length $=2.2-2.4$ ), and characterized by the total absence of denticles in the serration. The base of this tooth is relatively large and broad.

The species of Kalloconus and Trovaoconus exhibit essentially two kinds of radular morphologies (Additional file 1). The teeth in K. pulcher, and also in Trovaoconus trochulus, T. pseudonivifer and Trovaoconus atlanticoselvagem are narrow and elongated; the blade is moderately short being about one third to almost one-half the length of the anterior section of the tooth, which is distinctly longer than the posterior section of the tooth. There are many denticles (25 to 45 or more) in the long serration, arranged usually in multiple rows with a major row flanked by numerous smaller serrations. In the case of T. venulatus, Trovaoconus ateralbus, and Trovaoconus cf. ateralbus the teeth are broader, and the anterior and posterior sections are almost equal in length. There are 16 to 33 denticles in the serration, often coarse and hook-shaped in the middle portion, arranged initially in one row becoming two rows below.

\section{Dating of major cladogenetic events}

Major cladogenetic events within the reconstructed phylogeny were dated using an uncorrelated relaxed molecular clock model, which was calibrated using the age of Sal (28 mya; the oldest island of the archipelago) for the node separating Africonus from its sister group, L. ventricosus, and the age of the origins of São Vicente, Santo Antão, and Santa Luzia (7.5 mya) for the node splitting the lineage including the endemics to these islands from its sister group lineage including endemics to Maio and Santiago islands [24]. The first divergence event involving Kalloconus + Trovaoconus versus (paraphyletic) Lautoconus + Africonus was dated at 34 mya (Fig. 4; note that genera and species labels in the chronogram take into account proposed synonymizations, see Discussion). The divergence between the clade containing cones endemic to Senegal (+ L. guanche) and the clade including $L$. ventricosus plus Africonus was dated at 26 mya. The split between the latter two lineages was dated at 23 mya. The diversification of the crown group of Africonus into its four main lineages (I-IV) was estimated to have occurred between 9.4 - 6.9 mya (Fig. 4). The separation of Kalloconus and Trovaoconus was dated 


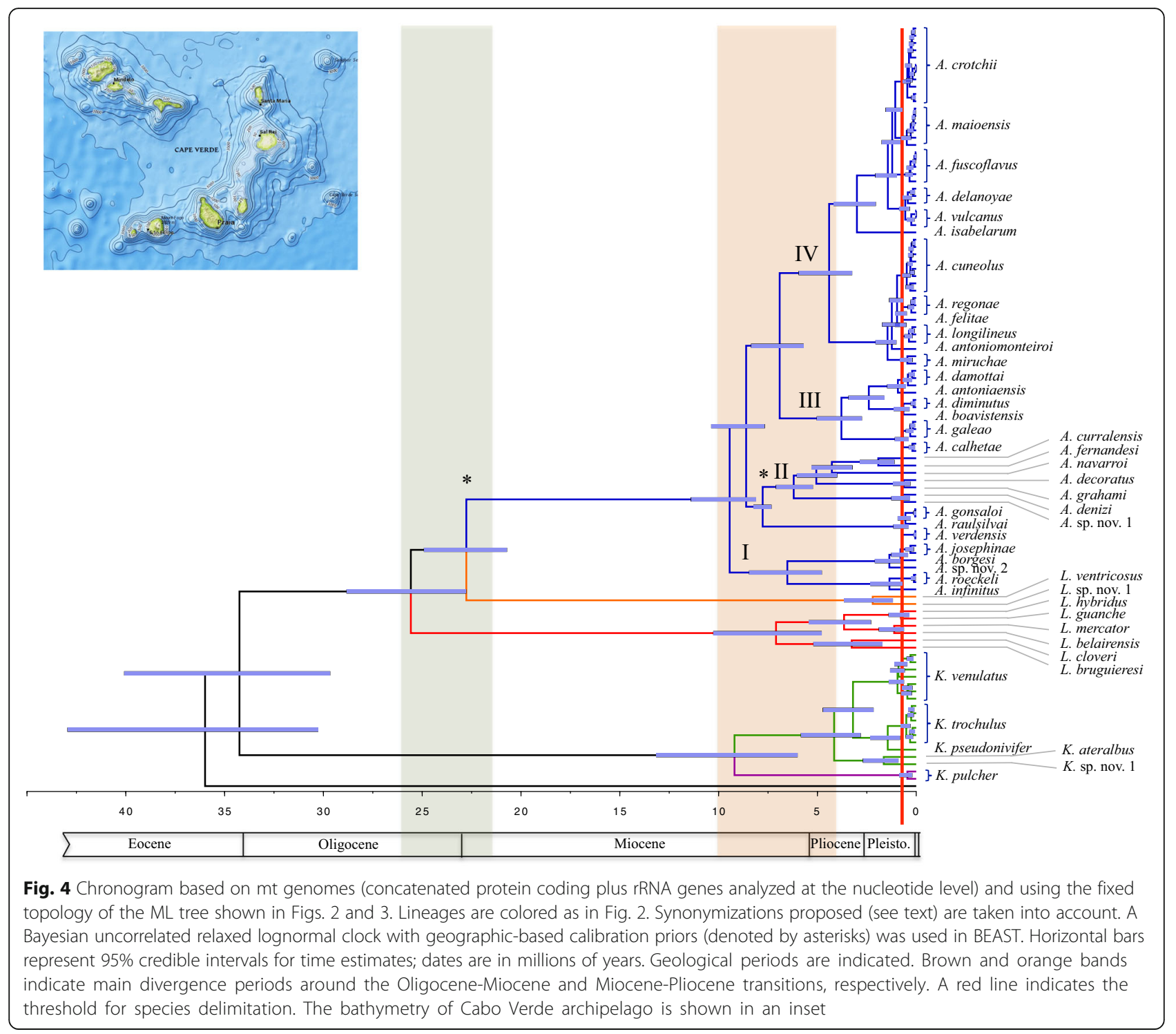

9 mya and the diversification of the crown group of Trovaoconus was established at 4 mya (Fig. 4).

\section{Diversification rates through time}

Variations in the diversification rates through time were estimated for Africonus and the hypothesis of a radiation during the evolutionary history of the clade was tested. The gamma-statistic, which measures departures from a constant rate of diversification, had values of $7.19(p<$ $0.05)$ and $3.12(\mathrm{p}<0.05)$ when considering the currently named (based on phenotypic traits) or only the hereproposed (considering genetic evidence) species for the genus, respectively. In both cases, the hypothesis of a radiation is accepted. According to the lineage through time plots (Fig. 5), the initial rate of increase in the number of species slowed down between six and one and a half million years ago regardless of the species delimitation hypothesis tested. Afterwards, the diversification rate accelerated considerably, and the increase in number of species either continued or abandoned a normal Yule process of speciation when considering the species delimitation hypothesis here proposed or the currently number of named species, respectively.

\section{Discussion}

Cone snails are marine gastropods well known to evolutionary biologists due to their extraordinary species and ecological diversity [25], but also to molecular biologists and pharmacologists due to their sophisticated venom cocktails [26], as well as to amateur naturalists due to their brightly colored and highly appreciated shells [27]. Therefore, they are the subject of intensive research across disciplines and additionally have received wide attention from the general public. There are more than 800 described species and this number increases steadily 

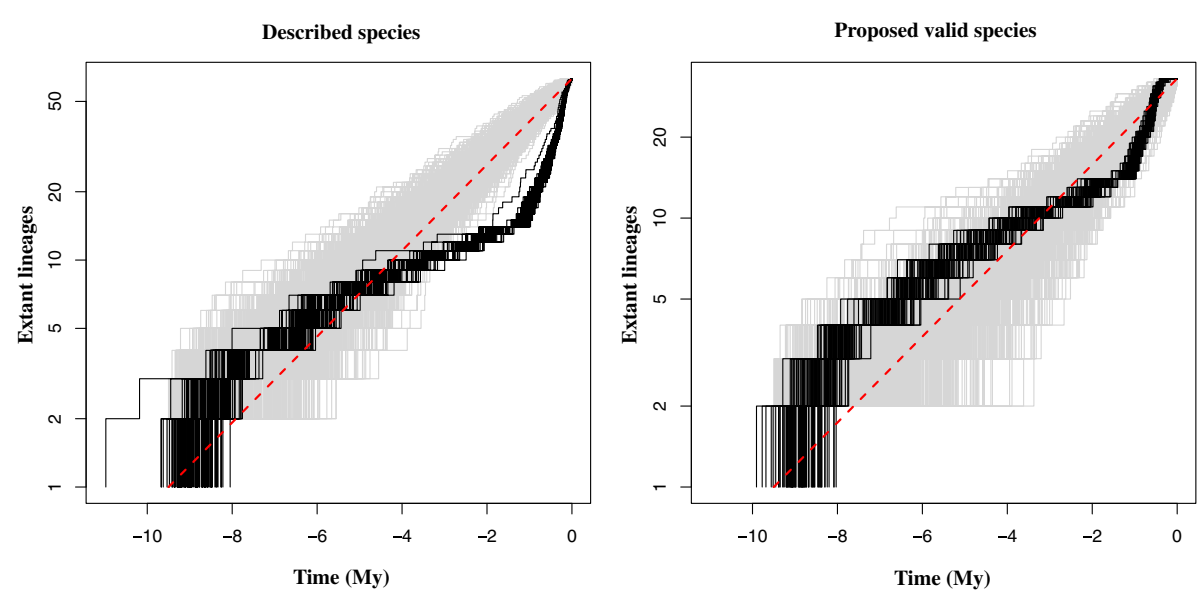

Fig. 5 Logarithmic lineage through time (LTT) plots of described (a) and proposed valid (b) species. The red bar indicates the pure Yule process of speciation, the grey shadow shows 1000 simulated trees and the black lines represent 100 trees randomly chosen among the trees generated by BEAST

every year [28]. Thus far, species description and identification of cones heavily relies on shell form, color and banding patterns, which may show great variety at local scale leading to important levels of synonymy within the family Conidae [7, 20]. In this regard, species delimitation could greatly improve with the aid of robust molecular phylogenies, which could be used in addition as framework to uncover the evolutionary patterns and processes underlying the diversification of the group. While reconstructing a robust phylogeny for all described cone species worldwide is cumbersome and at present unrealistic within the framework of a single study, it is possible, however, to accomplish a proof-of-concept study in a particular region [23].

We have here reconstructed a molecular phylogeny of cones endemic to Cabo Verde and allied species in the Macaronesian region, continental West Africa, and the Mediterranean region. These cones are particularly interesting from an evolutionary perspective as they have radiated in an oceanic archipelago and constitute a natural experiment to gain insights onto the processes governing diversification and adaptation [29]. Phylogenetic analyses were based on nearly complete $\mathrm{mt}$ genomes (only missing the control region and neighboring sequences) and included 105 specimens comprising most of the cone species diversity of the analyzed regions. Probabilistic methods of phylogenetic inference arrived at a robust and highly resolved phylogeny (virtually all nodes received high statistical support, which in most cases was maximal). To our knowledge, this is the first wide application of $\mathrm{mt}$ genomes to the resolution of a phylogeny within mollusks (but see $[30,31]$ for comparable examples in fish and insects, respectively). Previous studies in gastropods were restricted in the number of taxa analyzed (e.g., [22] for the family Conidae) but here we were able to achieve a lineage representation of the reference group (in this case, Cabo Verde cones) only previously attained by studies using few concatenated partial gene sequences (see e.g., [25] for the family Conidae or [1] for the cones of Cabo Verde). Previous phylogenetic studies using complete mt genomes have demonstrated that the level of resolution of these molecular markers is compromised above the superfamily level due to saturation, base composition biases, and among-lineage rate heterogeneity [32, 33]. Here, we show that phylogenetic performance of $\mathrm{mt}$ genomes achieves best results when analyzing closely related genera (and their corresponding species). Moreover, results were particularly promising taking into account that Africonus diversity in Cabo Verde was originated through radiation processes, which normally lead to relatively short tree nodes (often difficult to disentangle).

A thorough sampling of closely-related outgroup taxa allowed us to tackle key questions on the origin of the cones endemic to Cabo Verde and on their closest living sister groups. As previously reported, there are two independent origins of Cabo Verde cones, leading to the genera Africonus and Trovaoconus, respectively [1]. The closest living sister group of Africonus is L. ventricosus from the Mediterranean Sea and neighboring Atlantic Ocean. Therefore, the origin of this clade is clearly Macaronesian/Mediterranean and these cones are only distantly related to the geographically closer cones endemic to Senegal. These latter cones were ascribed to the genus Lautoconus (as was the case of L. guanche from Canary islands, deeply nested within the clade of Senegal cones; [23]). However, the closest sister group relationship of Africonus and $L$. ventricosus requires formal description of a different genus for Senegal cones (plus L. guanche), which will be done elsewhere. We could not include any representative of cones endemic to Angola (genus Varioconus) but a recent phylogeny based on partial coxl gene 
sequences recovered all these cones (including Varioconus jourdani from Saint Helena Island) as a monophyletic group sister to Senegal cones [34]. Alternatively, all previously mentioned genera could be merged into genus Lautoconus [35]. However, the relatively high levels of sequence divergence (using the sequence divergence of genus Chelyconus as reference) and the restricted (endemic) distribution of the clades fit better with the former taxonomic proposal. The closest living sister group of Trovaoconus is genus Kalloconus from West Africa. Therefore, the origin of this clade is clearly related to neighboring regions of the continent. Actually, the sequence divergence between Trovaoconus and Kalloconus is much lower than that estimated between Lautoconus and Africonus. This observation argues against maintaining the generic status of Trovaoconus, and supports the inclusion of their species within genus Kalloconus, as some authors have proposed [35]. Hence, Kalloconus would be a genus that is present throughout the coast of West Africa from Morocco to Angola as well as in Canary Islands and Cabo Verde.

Altogether, the two main clades in the reconstructed phylogeny show very distinct patterns of distribution. One clade includes a single genus with widespread distribution in Macaronesia and West Africa whereas the other clade, which occupies the same geographical regions, is divided into several valid genera (Africonus, Lautoconus, Varioconus, Gen. nov. for Senegal endemics). These distinct patterns could be explained partly taking into account differences in larval dispersal capabilities between the two clades [1]. According to the phylogeny, the ancestor of the Kalloconus clade was inferred to have planktotrophic larvae, capable of long dispersals whereas the ancestor of the other clade would have non-planktotrophic larvae, and thus a limited dispersal capability leading to restricted gene flow and higher rates of diversification [36, 37]. Interestingly, the ancestor of Kalloconus species endemic to Cabo Verde (former Trovaoconus species) lost planktotrophy, which is a common evolutionary pattern in insular species [38].

According to the reconstructed phylogeny, cones belonging to genus Africonus are divided into four main lineages (I-IV; with each further subdivided into two distinct clades). Species endemic to Maio and Boa Vista are found in all four lineages whereas species endemic to Sal form a clade within lineage IV, species from the westernmost islands (Santo Antão, São Vicente and Santa Luzia) form a clade within lineage II, and the single species from Santiago is recovered within lineage II. Unfortunately, we could not sample specimens of Africonus furnae from Brava and Africonus kersteni from São Nicolau, and cannot determine whether they could be ascribed to any of the above-mentioned four lineages or form their own independent lineages. The single origin of cones endemic to Sal, Santiago, and westernmost islands could be explained by the deep slopes separating these islands whereas the multiple origins of the cones found in Maio and Boa Vista could be associated to the relatively shallow seamount (Baixo João Valente) connecting both islands [1]. These differences in bathymetry in connection with past eustatic sea level changes could be determinant in preventing or promoting dispersal in Africonus species, whose larvae are all non-planktotrophic.

Diversification events among main lineages were concentrated in three major periods. The first one, around the Oligocene-Miocene boundary (23 mya), includes the divergence of cones endemic to Senegal (and Angola) from their sister clade, and the posterior separation within this sister clade of cones endemic to Cabo Verde and those endemic to the Mediterranean Sea and neighboring Atlantic Ocean. During Oligocene-Miocene transition, there was a global cooling event $[39,40]$, the ice sheet of Antarctica greatly expanded, and a sea level drop of $\sim 50 \mathrm{~m}$ occurred [41]. The second period corresponds to a sustained global cooling in the Late Miocene starting 12 mya [42] that produced an eustatic sea level drop between -10 and $-30 \mathrm{~m}$ from 6.26 to 5.50 Mya [43] and culminated with the Messinian Salinity crisis and the desiccation of the Mediterranean Sea at the end of the Miocene from 5.96 to 5.33 Mya [44]. During this period, the divergence of the main lineages within Africonus (I-IV), the cones endemic to Senegal, and Kalloconus occurred. Finally, a burst of speciation events is inferred during the Pleistocene when another cooling period characterized by extreme climate oscillations and drastic eustatic sea level changes concurring with glacial-interglacial periods [45]. Global cooling has been recently proposed to be a driver of diversification of marine species [46] in agreement with our results. The reconstructed phylogeny, the chronogram, and the current geographical distribution of the species altogether support that allopatry is the main mode of speciation for cone snails with non-planktotrophic larvae, as previously suggested [1]. The complex geology of the island of Boa Vista with several eruptions at $>16,15$ 12.5, and 9.5-4.5 mya [47], involving different parts of the island may have also contributed to creating additional niches along the coast and could explain that this island harbors the highest number of endemic cones.

The reconstructed phylogeny also allows inferring the evolution of the radula in the studied lineages $[8,48]$. All analyzed ingroup taxa are vermivorous [8]. Studies documenting potential specialization of the vermivore radular type to prey on specific worm species are scarce and restricted thus far to cone species preying on amphinomids [49]. Here, we show that most Africonus species show a "robust" radular type, which is shared also with $L$. ventricosus and a lineage of Senegal cones represented by Lautoconus cloveri and Lautoconus bruguieresi in the phylogeny [23]. Therefore, the common ancestor of cones endemic to Senegal (plus L. guanche), Africonus, and $L$. 
ventricosus was inferred to have a "robust" type radula. The "elongated" type of radular tooth, which was found in several species within lineage IV of Africonus, also appears in a lineage of Senegal cones that is represented by Lautoconus hybridus and L. guanche in the phylogeny [23]. The radular tooth of $A$. felitae resembles the "small" type observed in a lineage of Senegal cones represented by Lautoconus reticulatus and Lautoconus belairensis in the phylogeny [23]. The "broad" type of radular tooth that appeared independently in several lineages of Africonus has not been observed in any cone from Senegal. While shifts in radular type could be correlated with early cladogenesis in cones endemic to Senegal [23], the evolution of different types of radular tooth within Africonus was restricted to few specific cases. Thus, future studies are needed to determine whether in such cases there has been a dietary shift to prey on specific worms. The radula teeth identified in Kalloconus resemble the types "elongated" and "robust" observed within Lautoconus and Africonus, although are clearly distinct. This might indicate instances of convergence, and that only a discrete number of different main types of radula could be found in a given clade.

During the last few years, the number of new cone species described from Cabo Verde has increased at an astonishing rate (e.g., [12]). These new species are identified based on differences (often subtle) in shell shape and color, and their status needs to be contrasted with genetic data to uncover cases of local phenotypic variation within species due to either genetic polymorphism or phenotypic plasticity that may be producing overestimations of the number of species in the group [21]. In addition, genetic data could help identify cases of phenotypic convergence due to adaptation of genetically distinct populations (ecotypes) or species (sibling or cryptic) to similar environments [50-52], also affecting the total number of valid species. Comparative analyses of pairwise uncorrected sequence divergences taking into account the reconstructed phylogeny showed that some described species shared almost identical mt genomes with levels of sequence divergence normally considered to be associated to genetic variation at the population level. Clades comprising these sets of closely related sequences indicate that an uncorrected sequence divergence threshold around 1\% could be associated to the species status. This threshold lies well within the socalled grey zone of speciation between $0.5-2 \%$ [53]. Of course, these results need to be further confirmed with genomic nuclear data that discard potential events of incomplete lineage sorting and hybridization [54]. In addition, the present study could be further improved in the future by increasing the number of individuals analyzed per original species. Importantly, the comparative analyses on variation of diversification rates through time support the here proposed hypothesis of species delimitation as it concurs with a Yule process of speciation whereas the number of currently named species clearly exceed expectations and would imply an extraordinary recent acceleration of speciation rates.

Our study confirms the diversity of cone endemic to Cabo Verde but significantly reduces the number of valid species. Applying the threshold in a conservative manner (i.e., maintaining described species as valid in case of doubt due to closeness to the threshold) to cones endemic to Cabo Verde would reduce the number of valid species within the sampled Africonus from 65 to 32 (see Table 1 and Fig. 4). The proposed nomenclatural changes follow standard ICZN recommendations maintaining the most senior (oldest) name. Among the species not sampled, two correspond to São Nicolau and Brava islands, four of them are from the islands of São Vicente and Santa Luzía, and most likely represent valid species (Africonus bellulus, Africonus lugubris, Africonus saragasae, Africonus santaluziensis, A. kersteni and A. furnae) given the relative high sequence divergences found among species endemic to these islands. The 19 remaining ones were recently described, mostly from Boa Vista, and are expected to fall in most cases into some of the clades already discussed in the present work, and therefore may correspond to morphs of other described species. A direct consequence of synonymization is that some previously described species of rather restricted distribution are merged as populations into the new species, which considerably increase their range of distribution (Additional file 1). For instance, $A$. crotchii, which was reported as endemic from Southwest Boa Vista, would be now distributed also in the whole north half of the island. This increase in range of distribution of several species has important effects on their IUCN conservation status [3]. In the case of Kalloconus, some morphotypes attributed to Kalloconus pseudonivifer are now assigned to Kalloconus trochulus, and Kalloconus atlanticoselvagem is synonymized with $K$. trochulus. Our specimen of Kalloconus cf. byssinus is from North Senegal and has little sequence divergence compared to Kalloconus pulcher. In this case, it would be important to study $K$. byssinus from Mauritania or Morocco before considering synonymization. In the opposite direction, there are three clear instances of morphological convergence and thus, of the existence of cryptic species. Those are the cases of Africonus josephinae from Maio, Africonus cf. miruchae from São Vicente, and Kalloconus cf. ateralbus from Sal, which will be described as new species in due course.

\section{Conclusions}

We reconstructed a robust phylogeny based on mitochondrial genomes of cone snails endemic to Cabo Verde, which provides the necessary framework for future 
evolutionary studies focused on this radiation. The double origin of Cabo Verde endemic cones was supported. The ancestor of Africonus separated from L. ventricosus during the Oligocene-Miocene boundary (about 23 mya) and diversified into four main lineages (I to IV) in the Late Miocene (about 9.4-6.9 mya). The divergence of the ancestor of Kalloconus endemic to Cabo Verde from those inhabiting mainland occurred also in the Late Miocene whereas its diversification into three main lineages was dated in the Pliocene (4 mya). Main cladogenetic events within cones endemic to Cabo Verde coincide with global cooling periods, which were characterized by radical climate oscillations and eustatic sea level changes. Recurrent cycles of island connection/ disconnection likely favored speciation in allopatry in these cones, which lack a pelagic larval stage, and thus have limited dispersal capacity. Direct development evolved in the ancestor of Kalloconus endemic to Cabo Verde, likely associated to the colonization of the archipelago by a cone with a planktotrophic larval stage. However, in the case of Africonus, the ancestor that arrived to Cabo Verde was already non-planktotrophic as the corresponding independent evolutionary shift to direct development predated the separation of cones endemic to Senegal (and Canary Islands) from L. ventricosus plus Africonus. Radular types were modified during the diversification of Africonus from an ancestral "robust" type, although correlation with diet specializations await better knowledge of the specific worm species preyed by the different species of cones. Sequence divergence comparisons and reconstructed phylogenies supported the diversity of cone species endemic to Cabo Verde but significantly reduced its number, which was likely overestimated in the past due to important homoplasy in shell morphology, the, thus far, main discriminant character used for species description and identification.

\section{Methods}

\section{Samples and DNA extraction}

The complete list of specimens analyzed in this study corresponding to different populations and species of Africonus and Trovaoconus from Cabo Verde is shown in Table 1, as well as details on the respective sampling localities and museum vouchers. As outgroup taxa, we also sampled and analyzed one specimen of $L$. ventricosus from Formentera Island (Spain). Specimens were collected by snorkel at 1-3 m depth, or picked by hand at low tide. All samples were stored in $100 \%$ ethanol. The initial species identification (see corresponding column in Table 1) was based on comparison with type material (mostly deposited in the $\mathrm{MNCN}$ ) or consulting the original publications. Total DNA was isolated from 5 to $10 \mathrm{mg}$ of foot tissue following a standard phenol-chloroform extraction [55].

\section{Radular tooth preparation}

The radular sac was dissected from the main body and soft parts were digested in concentrated aqueous potassium hydroxide for $24 \mathrm{~h}$. The resulting mixture was then placed in a petri dish and examined with a binocular microscope. The entire radula was removed with fine tweezers and rinsed with distilled water, then mounted on a slide using Aquatex (Merck, Germany) mounting medium, and observed under a compound microscope. Photographs were taken with a charge-coupled device (CCD) camera attached to the microscope. Terminology for radular morphology follows [8], with abbreviations following [48]. Names of radular types follow [23].

\section{PCR amplification and sequencing}

Near-complete (without the control region) $\mathrm{mt}$ genomes were amplified through a combination of standard and long PCRs using the primers and following the protocols of [22]. Standard-PCR products were sequenced using Sanger technology. Long-PCR products were subjected to next-generation sequencing. Briefly, PCR amplified fragments from the same $\mathrm{mt}$ genome were pooled together in equimolar concentrations. For each cone mt genome a separate indexed library was constructed using the NEXTERA XT DNA library prep kit (Illumina, San Diego, CA, USA). The average size of the Nextera libraries varied between 307 and 345 bp. Libraries were pooled and run in an Illumina MiSeq platform (v. 2 chemistry; $2 \times 150$ pairedend) at Sistemas Genómicos (Valencia, Spain).

\section{Genome assembly and annotation}

The reads corresponding to each $\mathrm{mt}$ genome were sorted using the corresponding library indices, and read assembly was performed in the TRUFA webserver [56]. Briefly, adapters were removed using SeqPrep [57], quality of the reads was checked using FastQC v.0.10.1 [58], and raw sequences were trimmed and filtered out according to their quality scores using PRINSEQ v.0.20.3 [59]. Filtered reads were used for de novo assembly of each mt genome using default settings (minimum contig length: 200; sequence identity threshold: 0.95 ) of Trinity r2012-06-08 [60] in TRUFA, and only retaining contigs with a minimum length of $3 \mathrm{~kb}$. These contigs were used as starting point to assemble the $\mathrm{mt}$ genomes using Geneious $^{\circ}$ 8.0.3. First, the (raw) reads with a minimum identity of $99 \%$ were mapped against the contigs to correct possible sequence errors. Then, successive mapping iterations using a $100 \%$ identity as threshold were performed to elongate the contigs.

The $\mathrm{mt}$ genomes were annotated with the option "Annotate from Database" in Geneious" 8.0.3, using published $\mathrm{mt}$ genomes of Conidae as references. Annotations of the $13 \mathrm{mt}$ protein-coding genes were refined manually 
identifying the corresponding open reading frames using the invertebrate mitochondrial code. The transfer RNA (tRNA) genes were further identified with tRNAscan-SE 1.21 [61], which infer cloverleaf secondary structures (with a few exceptions that were determined manually). The ribosomal RNA (rRNA) genes were identified by sequence comparison with other Conidae mt genomes [22], and assumed to extend to the boundaries of adjacent genes [62]. GenBank accession numbers of each mt genome are provided in Table 1.

\section{Sequence alignment and phylogenetic analyses}

The newly sequenced $\mathrm{mt}$ genomes were aligned with the mt genomes of A. borgesi, Africonus infinitus, Africonus miruchae, T. ateralbus, T. pseudonivifer, T. venulatus, and C. ermineus from Cabo Verde, L. hybridus, L. mercator, $L$. belairensis, L. cloveri, L. bruguieresi, K. pulcher, and $K$. cf. byssinus from Senegal, L. guanche from Canary Islands, and $L$. ventricosus from Portugal, which were downloaded from GenBank (Table 1). A sequence data set was constructed concatenating the nucleotide sequences of the 13 $\mathrm{mt}$ protein-coding and two rRNA genes. The deduced amino acid sequences of the $13 \mathrm{mt}$ protein-coding genes were aligned separately and used to guide the alignment of the corresponding nucleotide sequences with Translator $\mathrm{X}$ [63]. Nucleotide sequences of the mt rRNA genes were aligned separately using MAFFT v7 [64] with default parameters. Ambiguously aligned positions were removed using Gblocks, v.0.91b [65] with the following settings: minimum sequence for flanking positions: $85 \%$; maximum contiguous non-conserved positions: 8 ; minimum block length: 10; gaps in final blocks: no. Finally, the different single alignments were concatenated using Geneious ${ }^{\circ}$ 8.0.3. Sequences where format converted for further analyses using the ALTER webserver [66]. The concatenated alignment is available at http://purl.org/phylo/treebase/ phylows/study/TB2:S21557.

Phylogenetic relationships were inferred using maximum likelihood (ML, [67]) and Bayesian inference (BI, [68]). For ML, we used RAxML v8.1.16 [69] with the rapid hill-climbing algorithm and 10,000 bootstrap pseudoreplicates (BP). BI analyses were conducted with MrBayes v3.1.2 [70], running four simultaneous Markov chains for 10 million generation, sampling every 1000 generations, and discarding the first $25 \%$ generations as burn-in (as judged by plots of ML scores and low SD of split frequencies) to prevent sampling before reaching stationarity. Two independent Bayesian inference runs were performed to increase the chance of adequate mixing of the Markov chains and to increase the chance of detecting failure to converge, as determined using Tracer v1.6 [71]. The effective sample size (ESS) of all parameters was checked to be above 200. Node support was assessed based on Bayesian Posterior Probabilities (BPP). A node was considered highly supported with BP and BPP values above $70 \%$ and 0.95 , respectively. The ML and BI phylogenetic trees are available at http://purl.org/phylo/treebase/phylows/study/TB2:S21557.

The best partition schemes and best-fit models of substitution for the data set were identified using PartitionFinder2 [72] with the Akaike information criterion [73]. For the protein-coding genes, the partitions tested were: all genes grouped; all genes separated (except atp6-atp8 and nad4-nad4L); and genes grouped by subunits ( $a t p, c o b, c o x$, and nad). In addition, these three partitions schemes were tested taking into account separately the three codon positions. The rRNA genes were tested with two different schemes, genes separated or combined.

\section{Estimation of divergence times}

The program BEAST v.1.8.0 [74] was used to perform a Bayesian estimation of divergence times. An uncorrelated relaxed molecular clock was used to infer branch lengths and nodal ages. The tree topology was fixed using the one recovered by the ML analysis. For the clock model, the lognormal relaxed-clock model was selected, which allows rates to vary among branches without any a priori assumption of autocorrelation between adjacent branches. For the tree prior, a Yule process of speciation was employed. Concatenated protein coding plus rRNA genes were analyzed at the nucleotide level. The partitions and models selected by PartitionFinder2 were applied (see results). The final Markov chain was run twice for 100 million generations, sampling every 10,000 generations, and the first 1000 trees were discarded as part of the burn-in process, according to the convergence of chains checked with Tracer v.1.5. [71]. The ESS of all parameters was above 200.

Despite the fact that there are many fossils of Conidae, it is difficult in many instances to be certain about species identifications given the important levels of homoplasy in shell shape [75]. Hence, although there are fossils attributed to L. ventricosus [76] and L. mercator [77], which could be applied to the reconstructed phylogeny, we opted to calibrate the clock using biogeographical events (i.e., the age of the islands of Cabo Verde). We run a preliminary analysis in which the posterior distribution of the estimated divergence times was obtained by specifying one calibration point as prior for the divergence time of the split between $L$. ventricosus and the genus Africonus. This genus is endemic to Cabo Verde, and we used the age of formation of the oldest island, Sal (28 Mya; [24]), as biogeographical calibration point. We applied a log-normal distribution as the prior model for the calibration and enforced the median 
divergence time to equal 25 (s.d. $=0.05$, offset $=0.7$ ). According to the results of the preliminary analysis, we found that only in the case of São Vicente, Santo Antão, and Santa Luzia, the early divergence of living cone endemic lineages followed the origin of the corresponding island, and therefore, we used a second calibration point corresponding to the origin of these islands about 7.5 mya [24]. We applied a log-normal distribution as the prior model for the calibration and enforced the median divergence time to equal 7.5 (s.d. $=0.03$, offset $=0)$. The BEAST tree is available at http:// purl.org/phylo/treebase/phylows/study/TB2:S21557.

\section{Diversification rate through time}

The chronogram was used to determine diversification rate through time of genus Africonus under alternative (phenotypic versus genetic) species delimitation hypotheses. A lineage through time (LTT) plot analysis was conducted using the APE $4.1 \mathrm{R}$ package [78]. A random sample of 100 trees was selected and mapped over a simulation of 1000 trees following a Yule process of speciation (net diversification rate $=0.4$ ). The phytools R package [79] was used to calculate the GammaStatistic [80].

\section{Additional file}

Additional file 1: Maps showing sampling localities; diversity of Kalloconus radular teeth. (ZIP 3996 kb)

\section{Acknowledgements}

We are indebted to Cabo Verde biology students Paulo Vasconcelos and Stiven Pires, as well as to our colleagues Sara Rocha, David Posada and Julio Rozas for their valuable help during sampling in Cabo Verde. We thank Dr. Rui Freitas from the Universidade de Cabo Verde (UniCV) for his continuous support and for granting us access to the facilities of the UniCV in Mindelo, São Vicente. We also thank Dr. Iderlindo Silva dos Santos and Dra. Sonia Monteiro de Pina Araujo from the Direcçao Nacional do Medio Ambiente of the Ministério do Ambiente, Habitação e Ordenamento do Território (MAHOT) of the Republic of Cabo Verde for their help with collecting permits (Autorizações 07/2013, 26/2013, 01/2104, 04/2015, and 03/2016). The sample of L. ventricosus from Formentera Island was obtained by Paula C. Rodríguez Flores. We are grateful to Jesús Marco and Aida Palacios, who provided access to the supercomputer Altamira at the Institute of Physics of Cantabria (IFCA-CSIC), member of the Spanish Supercomputing Network, for performing assembling and phylogenetic analyses.

\section{Funding}

This work was supported by the Spanish Ministry of Science and Innovation (CGL2013-45211-C2-2-P and CGL2016-75255-C2-1-P (AEI/FEDER, UE)) to RZ; BES-2011-051469 to JEU; BES-2014-069575 to SA; Doctorado Nacional-567, Colciencias-Universidad Nacional de Colombia to AME.

\section{Availability of data and materials}

Sequence data is available in GenBank.

\section{Authors' contributions}

MJT, CMLA, and RZ collected the material. MJT prepared the radula. SA, JEU, and AME generated the molecular data. SA analyzed the data. RZ wrote the first draft of the manuscript and all authors contributed to writing the final version. All authors read and approved the final manuscript.
Ethics approval and consent to participate

Not applicable.

\section{Consent for publication}

Not applicable.

\section{Competing interests}

The authors declare that they have no competing interests.

\section{Publisher's Note}

Springer Nature remains neutral with regard to jurisdictional claims in published maps and institutional affiliations.

\section{Author details}

${ }^{1}$ Museo Nacional de Ciencias Naturales (MNCN-CSIC), José Gutiérrez Abascal 2, 28006 Madrid, Spain. Departamento CMIM y Q. Inorgánica-INBIO, Facultad de Ciencias, Universidad de Cádiz, 11510 Puerto Real, Cádiz, Spain. ${ }^{3}$ Centre of Marine Sciences (CCMAR), Universidade do Algarve, Campus de Gambelas, 8005 - 139 Faro, Portugal.

Received: 15 June 2017 Accepted: 6 November 2017

Published online: 25 November 2017

References

1. Cunha RL, Castilho R, Rüber L, Zardoya R. Patterns of cladogenesis in the venomous marine gastropod genus Conus from the Cape Verde Islands. Syst Biol. 2005;54(4):634-50.

2. Duda TF, Rolán E. Explosive radiation of Cape Verde Conus, a marine species flock. Mol Ecol. 2005;14(1):267-72.

3. Peters $\mathrm{H}, \mathrm{O}^{\prime}$ Leary $\mathrm{BC}$, Hawkins JP, Roberts CM. The cone snails of Cape Verde: marine endemism at a terrestrial scale. Global Ecology and Conservation. 2016;7:201-13.

4. Tucker JK, Tenorio MJ. Illustrated catalog of the living cone shells. Wellington: MDM Publishing; 2013.

5. Olivera BM, Watkins M, Bandyopadhyay P, Imperial JS, de la Cotera EPH, Aguilar MB, Vera EL, Concepcion GP, Lluisma A. Adaptive radiation of venomous marine snail lineages and the accelerated evolution of venom peptide genes. Ann N Y Acad Sci. 2012;1267(1):61-70.

6. Cunha RL, Lima FP, Tenorio MJ, Ramos AA, Castilho R, Williams ST. Evolution at a different pace: distinctive phylogenetic patterns of cone snails from two ancient oceanic archipelagos. Syst Biol. 2014;63(6):971-87.

7. Cunha RL, Tenorio MJ, Afonso C, Castilho R, Zardoya R. Replaying the tape: recurring biogeographical patterns in Cape Verde Conus after 12 million years. Mol Ecol. 2008;17(3):885-901.

8. Tucker JK, Tenorio MJ. Systematic classification of recent and fossil conoidean gastropods: with keys to the genera of cone shells. Hackenheim: Conchbooks; 2009

9. Röckel D, Rolán E, Monteiro M. Cone shells from Cape Verde Islands - a difficult puzzle. I. Feito: Vigo, Spain; 1980.

10. Rolán E: Descripción de nuevas especies y subespecies del género Conus (Mollusca, Neogastropoda) para el archipiélago de Cabo Verde. Iberus 1990, Suppl. 2:5 - 70 .

11. Monteiro A, Tenorio MJ, Poppe GT. The family Conidae. The west African and Mediterranean species of Conus. A Conchological Iconography. Hackenheim: ConchBooks; 2004.

12. Cossignani T. Dieci nuovi coni da Capo Verde. Malacol Mostra Mond. 2014;82:18-29.

13. Cossignani T, Fiadeiro R. Cinque nuovi coni da Capo Verde. Malacol Mostra Mond. 2014:84:21-7.

14. Tenorio MJ, Afonso CML, Cunha RL, Rolán E. New species of Africonus (Gastropoda, Conidae) from boa vista in the Cape Verde archipelago: molecular and morphological characterization. Xenophora Taxonomy. 2014:2:5-21.

15. Afonso CML, Tenorio MJ. Recent findings from the islands of Maio and boa vista in the Cape Verde archipelago, West Africa: description of three new Africonus species (Gastropoda: Conidae). Xenophora Taxonomy. 2014;3:47-60.

16. Cossignani T, Fiadeiro R. Quattro nuovi coni da Capo Verde. Malacol Mostra Mond. 2014;83:14-9.

17. Cossignani T, Fiadeiro R. Otto nuovi coni da Capo Verde. Malacol Mostra Mond. 2017;94:26-36. 
18. Cossignani T, Fiadeiro R. Tre nuovi coni da Capo Verde. Malacol Mostra Mond. 2015;86:17-21.

19. Cossignani T, Fiadeiro R. Due nuovi coni da Capo Verde. Malacol Mostra Mond. 2015;87:3-5.

20. Duda TF, Palumbi SR. Developmental shifts and species selection in gastropods. Proc Natl Acad Sci U S A. 1999;96(18):10272-7.

21. Dayrat B. Towards integrative taxonomy. Biol J Linn Soc. 2005;85(3):407-15.

22. Uribe JE, Puillandre N, Zardoya R. Beyond Conus: phylogenetic relationships of Conidae based on complete mitochondrial genomes. Mol Phylogenet Evol. 2017;107:142-51.

23. Abalde S, Tenorio MJ, CML A, Zardoya R. Mitogenomic phylogeny of cone snails endemic to Senegal. Mol Phylogenet Evol. 2017;112:79-87.

24. Holm PM, Grandvuinet T, Friis J, Wilson JR, Barker AK, Plesner S. An 40Ar-39Ar study of the Cape Verde hot spot: temporal evolution in a semistationary plate environment. J. Geophys. Res. Solid Earth. 2008;113(B8):n/a-n/a.

25. Puillandre N, Bouchet P, Duda TF Jr, Kauferstein S, Kohn AJ, Olivera BM, Watkins M, Meyer C. Molecular phylogeny and evolution of the cone snails (Gastropoda, Conoidea). Mol Phylogenet Evol. 2014;78:290-303.

26. Olivera BM. Conus peptides: biodiversity-based discovery and exogenomics. J Biol Chem. 2006;281(42):31173-7.

27. Filmer RM. A catalogue of nomenclature and taxonomy in the living Conidae 1758 - 1998. Leiden: Backhuys Publishers; 2001.

28. Bouchet P, Gofas S. Conidae Fleming, 1822. In: MolluscaBase (2017). 2010. Accessed through: World Register of Marine Species at https:// marinespeciesorg/aphiaphp?p=taxdetails\&id=14107. Accessed 11 Nov 2017.

29. Warren BH, Simberloff D, Ricklefs RE, Aguilée R, Condamine FL, Gravel D, Morlon H, Mouquet N, Rosindell J, Casquet J, et al. Islands as model systems in ecology and evolution: prospects fifty years after MacArthur-Wilson. Ecol Lett. 2015;18(2):200-17.

30. Crampton-Platt A, Timmermans MJTN, Gimmel ML, Kutty SN, Cockerill TD, Vun Khen C, Vogler AP. Soup to tree: the phylogeny of beetles inferred by mitochondrial metagenomics of a Bornean rainforest sample. Mol Biol Evol. 2015;32(9):2302-16.

31. Saitoh K, Sado T, Mayden RL, Hanzawa N, Nakamura K, Nishida M, Miya M. Mitogenomic evolution and interrelationships of the Cypriniformes (Actinopterygii: Ostariophysi): the first evidence toward resolution of higherlevel relationships of the world's largest freshwater fish clade based on 59 whole mitogenome sequences. J Mol Evol. 2006;63(6):826-41.

32. Stöger I, Schrödl M. Mitogenomics does not resolve deep molluscan relationships (yet?). Mol Phylogenet Evol. 2013;69(2):376-92.

33. Osca D, Irisarri I, Todt C, Grande C, Zardoya R. The complete mitochondrial genome of Scutopus ventrolineatus (Mollusca: Chaetodermomorpha) supports the Aculifera hypothesis. BMC Evol Biol. 2014;14(1):1-10.

34. Tenorio MJ, Lorenz F, Dominguez M. New insights into Conus jourdani da Motta, 1984 (Gastropoda, Conidae), an endemic species from Saint Helena Island. Xenophora Taxonomy. 2016;11:32-42.

35. Puillandre N, Duda TF, Meyer C, Olivera BM, Bouchet P. One, four or 100 genera? A new classification of the cone snails. J Molluscan Stud. 2015;81:1-23.

36. Jablonski D. Larval ecology and macroevolution in marine invertebrates. Bull Mar Sci. 1986;39(2):565-87.

37. Scheltema RS. Planktonic and non-planktonic development among prosobranch gastropods and its relationship to the geographic range of species. In: Ryland JS, Tyler RA, editors. Reproduction, genetics and distribution of marine organisms. Fredensborg: Olsen \& Olsen; 1989. p. 183-8.

38. Swearer SE, Shima JS, Hellberg ME, Thorrold SR, Jones GP, Robertson DR Morgan SG, Selkoe KA, Ruiz GM, Warner RR. Evidence of self-recruitment in demersal marine populations. Bull Mar Sci. 2002;70(1):251-71.

39. Miller KG, Kominz MA, Browning JV, Wright JD, Mountain GS, Katz ME, Sugarman PJ, Cramer BS, Christie-Blick N, Pekar SF. The Phanerozoic record of global sea-level change. Science. 2005;310(5752):1293.

40. Zachos JC, Flower BP, Paul H. Orbitally paced climate oscillations across the Oligocene/Miocene boundary. Nature. 1997;388(6642):567-70.

41. Beddow HM, Liebrand D, Sluijs A, Wade BS, Lourens LJ. Global change across the Oligocene-Miocene transition: high-resolution stable isotope records from IODP site U1334 (equatorial Pacific Ocean). Paleoceanography. 2016;31(1):81-97.

42. Herbert TD, Lawrence KT, Tzanova A, Peterson LC, Caballero-Gill R, Kelly CS. Late Miocene global cooling and the rise of modern ecosystems. Nat Geosci. 2016;9(11):843-7.
43. Hodell DA, Curtis JH, Sierro FJ, Raymo ME. Correlation of late Miocene to early Pliocene sequences between the Mediterranean and North Atlantic. Paleoceanography. 2001;16(2):164-78.

44. Krijgsman W, Hilgen FJ, Raffi I, Sierro FJ, Wilson DS. Chronology, causes and progression of the Messinian salinity crisis. Nature. 1999;400(6745):652-5.

45. Lisiecki LE, Raymo ME. A Pliocene-Pleistocene stack of 57 globally distributed benthic $\delta 180$ records. Paleoceanography. 2005;20:PA1003.

46. Davis KE, Hill J, Astrop TI, Wills MA. Global cooling as a driver of diversification in a major marine clade. Nat Commun. 2016;7:13003.

47. Dyhr CT, Holm PM. A volcanological and geochemical investigation of boa vista, Cape Verde Islands; $40 \mathrm{Ar} / 39 \mathrm{Ar}$ geochronology and field constraints. J Volcanol Geotherm Res. 2010;189(1-2):19-32.

48. Kohn AJ, Nishi M, Pernet B. Snail spears and scimitars: a character analysis of Conus radular teeth. J Molluscan Stud. 1999;65:461-81.

49. Duda TF, Kohn AJ, Palumbi SR. Origins of diverse feeding ecologies within Conus, a genus of venomous marine gastropods. Biol J Linn Soc. 2001;73(4):391-409.

50. Dowle EJ, Morgan-Richards M, Brescia F, Trewick SA. Correlation between shell phenotype and local environment suggests a role for natural selection in the evolution of Placostylus snails. Mol Ecol. 2015;24(16):4205-21.

51. Hollander J, Butlin RK. The adaptive value of phenotypic plasticity in two ecotypes of a marine gastropod. BMC Evol Biol. 2010;10(1):333.

52. Knowlton N. Sibling species in the sea. Annu Rev Ecol Syst. 1993;24(1):189-216.

53. Roux C, Fraïsse C, Romiguier J, Anciaux Y, Galtier N, Bierne N. Shedding light on the Grey zone of speciation along a continuum of genomic divergence. PLoS Biol. 2016;14(12):e2000234.

54. Gerard D, Gibbs HL, Kubatko L. Estimating hybridization in the presence of coalescence using phylogenetic intraspecific sampling. BMC Evol Biol. 2011;11(1):291.

55. Sambrook J, Fritsch EF, Maniatis T. Molecular cloning: a laboratory manual. 2nd ed. New York: Cold Spring Harbor Laboratory Press; 1989.

56. Kornobis E, Cabellos L, Aguilar F, Frías-López C, Rozas J, Marco J, Zardoya R. TRUFA: a user-friendly web server for de novo RNA-seq analysis using cluster computing. Evol Bioinforma. 2015; 11 (Supplementary Material 23873):97-104.

57. StJohn J. SeqPrep. 2011. https://githubcom/stjohn/SeqPrep.

58. Andrews S. FastQC. 2010. http://wwwbioinformaticsbabrahamacuk/projects/ fastac/.

59. Schmieder R, Edwards R. Quality control and preprocessing of metagenomic datasets. Bioinformatics. 2011;27(6):863-4.

60. Grabherr MG, Haas BJ, Yassour M, Levin JZ, Thompson DA, Amit I, Adiconis X, Fan L, Raychowdhury R, Zeng Q, et al. Full-length transcriptome assembly from RNA-Seq data without a reference genome. Nat Biotech. 2011;29(7):644-52.

61. Schattner P, Brooks AN, Lowe TM. The tRNAscan-SE, snoscan and snoGPS web servers for the detection of tRNAs and snoRNAs. Nucleic Acids Res. 2005;33(suppl 2):W686-9.

62. Boore JL, Macey JR, Medina M. Sequencing and comparing whole mitochondrial genomes of animals. Methods Enzymol. 2005;395:311-48.

63. Abascal F, Zardoya R, Telford MJ. TranslatorX: multiple alignment of nucleotide sequences guided by amino acid translations. Nucleic Acids Res. 2010;38(Web Server issue):W7-13.

64. Katoh K, Standley DM. MAFFT multiple sequence alignment software version 7: improvements in performance and usability. Mol Biol Evol. 2013; 30(4):772-80.

65. Castresana J. Selection of conserved blocks from multiple alignments for their use in phylogenetic analysis. Mol Biol Evol. 2000;17(4):540-52.

66. Glez-Peña D, Gómez-Blanco D, Reboiro-Jato M, Fdez-Riverola F, Posada D. ALTER: program-oriented conversion of DNA and protein alignments. Nucleic Acids Res. 2010;38(suppl 2):W14-8.

67. Felsenstein J. Evolutionary trees from DNA sequences: a maximum likelihood approach. J Mol Evol. 1981;17(6):368-76.

68. Huelsenbeck J, Ronquist F. MrBayes: Bayesian inference of phylogenetic trees. Bioinformatics. 2001;17:754-5.

69. Stamatakis A. RAxML-VI-HPC: maximum likelihood-based phylogenetic analyses with thousands of taxa and mixed models. Bioinformatics. 2006; 22(21):2688-90.

70. Ronquist F, Huelsenbeck JP. MrBayes 3: Bayesian phylogenetic inference under mixed models. Bioinformatics. 2003;19(12):1572-4.

71. Rambaut A, Drummond AJ. Tracer v1.4. 2007. Available from http://beast. bio.ed.ac.uk/Tracer. 
72. Lanfear R, Frandsen PB, Wright AM, Senfeld T, Calcott B. PartitionFinder 2: new methods for selecting partitioned models of evolution for molecular and morphological phylogenetic analyses. Mol Biol Evol. 2017:34(3):772-3.

73. Akaike $\mathrm{H}$. Information theory and an extension of the maximum likelihood principle. In: Petrov BN, Csaki F, editors. 2nd international symposium on information theory. Budapest: Akademiai Kiado; 1973. p. 267-81.

74. Drummond A, Rambaut A. BEAST: Bayesian evolutionary analysis by sampling trees. BMC Evol Biol. 2007;7:214.

75. Duda TF, Bolin MB, Meyer CP, Kohn AJ. Hidden diversity in a hyperdiverse gastropod genus: discovery of previously unidentified members of a Conus species complex. Mol Phylogenet Evol. 2008;49(3):867-76.

76. Sacco F: I molluschi dei terreni terziarii del Piemonte e della Liguria. Conidae e Conorbidae, vol. XIII. Torino. Italy: Stamperia Reale; 1893.

77. Glibert M. Les Conacea fossiles du Cénozoïque étranger des collections de I'nstitut Royal des Sciences Naturelles de Belgique. Institut Royal des Sciences Naturelles de Belgique Mémoire, série 2. 1960;64:1-132.

78. Paradis E, Claude J, Strimmer K. APE: analyses of phylogenetics and evolution in R language. Bioinformatics. 2004;20(2):289-90.

79. Revell L. Phytools: an R package for phylogenetic comparative biology (and other things). Methods Ecol Evol. 2012;3(2):217-23.

80. Pybus OG, Harvey PH. Testing macro-evolutionary models using incomplete molecular phylogenies. Proc R Soc Lond B Biol Sci. 2000;267(1459):2267.

\section{Submit your next manuscript to BioMed Central and we will help you at every step:}

- We accept pre-submission inquiries

- Our selector tool helps you to find the most relevant journal

- We provide round the clock customer support

- Convenient online submission

- Thorough peer review

- Inclusion in PubMed and all major indexing services

- Maximum visibility for your research

Submit your manuscript at www.biomedcentral.com/submit 